\title{
Relativistic simulations of rotational core collapse II. Collapse dynamics and gravitational radiation
}

\author{
H. Dimmelmeier ${ }^{1}$, J. A. Font ${ }^{1,2}$, and E. Müller ${ }^{1}$ \\ 1 Max-Planck-Institut für Astrophysik, Karl-Schwarzschild-Str. 1, 85741 Garching, Germany \\ ${ }^{2}$ Departamento de Astronomía y Astrofísica, Universidad de Valencia, 46100 Burjassot (Valencia), Spain
}

Received 12 April 2002 / Accepted 4 July 2002

\begin{abstract}
We have performed hydrodynamic simulations of relativistic rotational supernova core collapse in axisymmetry and have computed the gravitational radiation emitted by such an event. The Einstein equations are formulated using the conformally flat metric approximation, and the corresponding hydrodynamic equations are written as a first-order flux-conservative hyperbolic system. Details of the methodology and of the numerical code have been given in an accompanying paper. We have simulated the evolution of 26 models in both Newtonian and relativistic gravity. The initial configurations are differentially rotating relativistic 4/3-polytropes in equilibrium which have a central density of $10^{10} \mathrm{~g} \mathrm{~cm}^{-3}$. Collapse is initiated by decreasing the adiabatic index to some prescribed fixed value. The equation of state consists of a polytropic and a thermal part for a more realistic treatment of shock waves. Any microphysics like electron capture and neutrino transport is neglected. Our simulations show that the three different types of rotational supernova core collapse and gravitational waveforms identified in previous Newtonian simulations (regular collapse, multiple bounce collapse, and rapid collapse) are also present in relativistic gravity. However, rotational core collapse with multiple bounces is only possible in a much narrower parameter range in relativistic gravity. The relativistic models cover almost the same range of gravitational wave amplitudes $\left(4 \times 10^{-21} \leq h^{\mathrm{TT}} \leq 3 \times 10^{-20}\right.$ for a source at a distance of $10 \mathrm{kpc}$ ) and frequencies $(60 \mathrm{~Hz} \leq v \leq 1000 \mathrm{~Hz})$ as the corresponding Newtonian ones. Averaged over all models, the total energy radiated in the form of gravitational waves is $8.2 \times 10^{-8} M_{\odot} c^{2}$ in the relativistic case, and $3.6 \times 10^{-8} M_{\odot} c^{2}$ in the Newtonian case. For all collapse models that are of the same type in both Newtonian and relativistic gravity, the gravitational wave signal is of lower amplitude. If the collapse type changes, either weaker or stronger signals are found in the relativistic case. For a given model, relativistic gravity can cause a large increase of the characteristic signal frequency of up to a factor of five, which may have important consequences for the signal detection. Our study implies that the prospects for detection of gravitational wave signals from axisymmetric supernova rotational core collapse do not improve when taking into account relativistic gravity. The gravitational wave signals obtained in our study are within the sensitivity range of the first generation laser interferometer detectors if the source is located within the Local Group. An online catalogue containing the gravitational wave signal amplitudes and spectra of all our models is available at the URL http://www . mpa-garching.mpg.de/Hydro/hydro.html.
\end{abstract}

Key words. gravitation - gravitational waves - hydrodynamics - stars: neutron - stars: rotation - stars: supernovae: general

\section{Introduction}

At the end of their thermonuclear evolution, massive stars develop a core composed of iron group nuclei (hence iron core) which becomes dynamically unstable against gravitational collapse. The iron core collapses to a neutron star or a black hole, releasing gravitational binding energy of the order $\sim 3 \times$ $10^{53} \operatorname{erg}\left(M / M_{\odot}\right)^{2}(R / 10 \mathrm{~km})^{-1}$, which is sufficient to power a supernova explosion. If the core collapse and/or the supernova explosion are nonspherical, part of the liberated gravitational binding energy will be emitted in the form of gravitational waves. Nonsphericity can be caused by the effects of rotation, convection and anisotropic neutrino emission leading either to

Send offprint requests to: $\mathrm{H}$. Dimmelmeier, e-mail: harrydee@mpa-garching .mpg.de a large-scale deviation from spherical symmetry or to smallscale statistical mass-energy fluctuations (for a review, see e.g., Müller 1998).

According to present knowledge the energy radiated away in form of gravitational waves does not exceed $10^{-6} M_{\odot} c^{2}$ in rotational core collapse (Müller 1982; Finn \& Evans 1990; Mönchmeyer et al. 1991; Bonazzola \& Marck 1993; Yamada \& Sato 1994a; Zwerger \& Müller 1997; Rampp et al. 1998; Fryer et al. 2002) and from convection or anisotropic neutrino emission in neutrino-driven supernovae (Müller \& Janka 1997). The frequency of the emitted radiation ranges from about a few $\mathrm{Hz}$ to a few $\mathrm{kHz}$, and the (dimensionless) signal amplitudes for a source located at a distance of $10 \mathrm{Mpc}$ (within the Virgo cluster) do not exceed $\sim 10^{-22}$. The smallness of the signals calls for accurate waveform templates to alleviate the technical 
difficulties in extracting the signal from noisy data (Pradier et al. 2001). For the current generation of laser interferometric detectors (GEO 600, LIGO, VIRGO, TAMA) such small amplitudes imply that the prospects for detection of gravitational waves from core collapse supernovae are limited to those events occurring within the Local Group. However, if measured, a gravitational wave signal does provide (as observations of neutrinos) a direct diagnosis of the dynamics of the events.

Investigations of rotational core collapse are also important in the context of the supernova explosion mechanism (Müller \& Hillebrandt 1981; Bodenheimer \& Woosley 1983; Symbalisty 1984; Mönchmeyer \& Müller 1989; Janka \& Mönchmeyer 1989; Imshennik \& Nadezhin 1992; Yamada \& Sato 1994b; Fryer \& Heger 2000) and for the collapsar scenario of gammaray bursts, where the collapse of a rotating massive star leads to the formation of a Kerr black hole and a relativistic jet (MacFadyen et al. 2001; Aloy et al. 2000; Wheeler et al. 2000).

Numerous studies have addressed quite different aspects of rotational core collapse at different levels of sophistication. Ideally, such studies should incorporate a relativistic treatment of gravity and hydrodynamics without symmetry restrictions, a detailed description of the complex microphysics and of the neutrino transport, and should use consistent initial models from evolutionary calculations of rotating stars. The latter point is still a nagging problem (for a major step forward in this context see Heger et al. 2000), as up to now all studies, except that of Fryer et al. (2002), have relied on parameterized initial models.

As the general relativistic corrections of the gravitational potential during supernova core collapse, and for a neutron star, do not exceed $30 \%$, it is often argued that they can be neglected or only taken into account approximately. While this approach may be justified to some degree in the case of non-rotational core collapse, it becomes very questionable when the core possesses a significant amount of angular momentum. In the latter case, a relativistic treatment of gravity is much more important, because the stabilizing effect of rotation is counteracted by the destabilizing effect of the deeper relativistic potential. This is reflected in the expression for the critical adiabatic index $\gamma_{\text {crit }}$, below which a rigidly rotating relativistic configuration is dynamically unstable against pseudo-radial (linear) isentropic perturbations (see, e.g., Chap. 14 of Tassoul 1978):

$\gamma_{\text {crit }}=\frac{4}{3}-\frac{2}{9} \frac{\Omega^{2} I}{\left|E_{\text {pot }}\right|}+k \frac{R_{\mathrm{S}}}{R}$.

Here $\Omega, I, E_{\mathrm{pot}}, R_{\mathrm{S}}$, and $R$ are the (constant) angular velocity, the moment of inertia about the center of mass, the gravitational potential energy, the Schwarzschild radius, and the radius of the star, respectively. The positive constant $k$ depends on the density distribution of the star.

Axisymmetric Newtonian hydrodynamic collapse and explosion simulations using a realistic equation of state (EoS) and some treatment of weak interaction processes have been performed by Müller (1982), Bodenheimer \& Woosley (1983), Symbalisty (1984) and Bonazzola \& Marck (1993) neglecting neutrino transport, and by Mönchmeyer \& Müller (1989), Janka \& Mönchmeyer (1989), Mönchmeyer et al. (1991), Imshennik \& Nadezhin (1992), Fryer \& Heger (2000) and
Fryer et al. (2002) employing some approximative description of neutrino transport. In addition, Finn \& Evans (1990), Yamada \& Sato (1994b) and Zwerger \& Müller (1997) have performed Newtonian parameter studies of the axisymmetric collapse of rotating polytropes. Rampp et al. (1998) and Brown (2001) have extended the work of Zwerger \& Müller (1997) by relaxing the assumption of axisymmetry.

Wilson (1979), Evans (1986), Nakamura (1981, 1983) and Stark \& Piran (1985) pioneered investigations of axisymmetric collapse of rotating configurations in full general relativity (GR). Wilson (1979) computed neutron star bounces of $\gamma=$ 2 polytropes, while Nakamura (1981) (see also Nakamura et al. 1987) simulated the formation of rotating black holes resulting from the collapse of a $10 M_{\odot}$ "core" of a massive star with different amounts of rotational energy and an initial central density of $3 \times 10^{13} \mathrm{~g} \mathrm{~cm}^{-3}$. Nakamura (1983) (see also Nakamura et al. 1987) considered a configuration consisting of a neutron $\operatorname{star}\left(M=1.09 M_{\odot}, \rho_{\mathrm{c}}=10^{15} \mathrm{~g} \mathrm{~cm}^{-3}\right)$ with an accreted envelope of $0.81 M_{\odot}$, which was thought to mimic mass fall-back in a supernova explosion. To this configuration he added rotation and infall velocity, and simulated the evolution depending on the prescribed rotation rates and rotation laws. In both scenarios the EoS consisted of a relativistic degenerate lepton gas $(\gamma=4 / 3)$ at low densities $\left(\rho \leq \rho^{*} \equiv 3 \times 10^{14} \mathrm{~g} \mathrm{~cm}^{-3}\right.$, and of a stiff $(\gamma=2)$ component at large densities $\left(\rho>\rho^{*}\right)$. Stark \& Piran (1985) were the first to compute the gravitational radiation from the relativistic collapse of a rotating polytropic $(\gamma=2)$ star to a black hole. The initial model was a spherically symmetric relativistic polytrope in equilibrium of mass $M$, central density $1.9 \times 10^{15}\left(M / M_{\odot}\right)^{-2}$, and radius $6 G M / c^{2}=8.8 \times 10^{5} M / M_{\odot} \mathrm{cm}$. Rotational collapse was induced by lowering the pressure in the initial model by a prescribed fraction, and by simultaneously adding an angular momentum distribution approximating rigid-body rotation. Stark $\&$ Piran (1985) found a low efficiency of gravitational wave emission $\left(E_{\mathrm{rad} \text { tot }} / M c^{2}<7 \times 10^{-4}\right.$, where $E_{\text {rad tot }}$ is the total energy radiated by graviational waves), and that for sufficient rotation the star bounces and no black hole forms.

After the work of Stark \& Piran (1985) it took about 15 years before the next simulations of GR rotational core collapse were published. This was mainly caused by persistent numerical problems occurring in axisymmetric GR simulations due to coordinate singularities at the symmetry (= rotation) axis. These coordinate singularities hamper the development of methods which guarantee accuracy and stability in long term (covering many dynamical time scales) simulations. Alcubierre et al. (2001) proposed a method which does not suffer from stability problems and where, in essence, Cartesian coordinates are used even for axisymmetric systems. Using this method Shibata (2000) investigated the effects of rotation on the criterion for prompt adiabatic collapse of rigidly and differentially rotating $(\gamma=2)$ polytropes to a black hole. Collapse of the initial approximate (computed by assuming a conformally flat spatial metric) equilibrium models was induced by a pressure reduction. Shibata (2000) found that the criterion for black hole formation depends strongly on the amount of angular momentum, but only weakly on its (initial) distribution. He also studied the effects of shock heating using a gamma-law EoS, and 
found that shock heating is important in preventing prompt collapse to black holes in case of large rotation rates.

Hayashi et al. (1999) investigated the possibility of secular, i.e. quasi-static, core contraction from white dwarf to neutron star densities using equilibrium sequences of rapidly rotating, general relativistic compact stars with phenomenological equations of state. They demonstrated that there is a possibility for the existence of "fizzlers" in the framework of GR, at least for the simplified equations of state used in their study.

The above discussion shows that previous investigations in GR rotational core collapse were mainly concerned with the question of black hole formation under idealized conditions, but none of these studies has really addressed the problem of supernova core collapse which proceeds from white dwarf densities to neutron star densities, involves core bounce, shock formation, and shock propagation. Exactly this is the motivation for the present work. To this end, we apply the numerical methodology presented in an accompanying paper (Dimmelmeier et al. 2002, hereafter Paper I) and study the dynamics of axisymmetric, relativistic rotational supernova core collapse and the associated emission of gravitational radiation. In particular, we have simulated the collapse of a comprehensive set of rotating relativistic stellar models parameterized by the initial degree of differential rotation, the initial rotation rate and the adiabatic indices at subnuclear (supranuclear) matter densities. Thus, our study is a GR extension of the previous work of Zwerger (1995) and Zwerger \& Müller (1997), who computed the gravitational radiation from rotational core collapse supernovae using Newtonian gravity. First results from our study have already been published in a short communication (Dimmelmeier et al. 2001) ${ }^{1}$.

The organization of the paper is as follows: Sect. 2 contains a brief repetition of the assumptions made in our study (for more details see Paper I). In Sect. 3 we analyze in detail the collapse dynamics and introduce the three different types of collapse and gravitational waveforms we have identified. Section 4 is devoted to the gravitational wave emission of the models, and to the prospects of detectability of gravitational waves from core collapse supernovae. We conclude with a summary in Sect. 5. Additional information is given in Appendix A where we discuss the issue of gravitational wave extraction.

\section{Model assumptions}

The matter model obeys an ideal gas EoS with the pressure $P$ consisting of a polytropic and a thermal part for a more realistic treatment of the effects of shock waves (Janka et al. 1993; Zwerger \& Müller 1997). Since we are mostly interested in the gravitational radiation emission, which is controlled by the bulk motion of the fluid, we neglect microphysics like electron capture and neutrino transport. The initial configurations are differentially rotating relativistic 4/3-polytropes in equilibrium (Komatsu et al. 1989a,b; Stergioulas \& Friedman 1995)

\footnotetext{
1 Note that due to a programming error, which we found only after the Letter was published, the values of some quantities (e.g., the bounce densities and maximum wave amplitudes) given in the Letter differ from those presented here, but all qualitative results remain unchanged.
}

which are marginally stable, and which have a central density $\rho_{\text {cini }}=10^{10} \mathrm{~g} \mathrm{~cm}^{-3}$. Collapse is initiated by decreasing the adiabatic index (initially $4 / 3$ in all models) to some prescribed fixed value $\gamma_{1}$ (for more details see Paper I).

The initial models are determined by three parameters, which are also used to name the models. The first parameter $A$ is a length scale, which specifies the degree of differential rotation (see Eq. (33) of Paper I). The smaller the value of $A$ the more differentially rotating the model is. Model parameters A1, A2, A3 and A4 correspond to $A=5 \times 10^{9} \mathrm{~cm}, A=10^{8} \mathrm{~cm}, A=$ $5 \times 10^{7} \mathrm{~cm}$, and $A=10^{7} \mathrm{~cm}$, respectively. The second parameter is the initial rotation rate $\beta_{\text {rot ini }}$, which is given by the ratio of rotational energy and the absolute value of the gravitational binding energy. Model parameters B1, B2, B3, B4 and B5 correspond to $\beta_{\text {rot ini }}=0.25 \%, 0.5 \%, 0.9 \%, 1.8 \%$ and $4 \%$, respectively. The third parameter $\gamma_{1}$ is the adiabatic index at subnuclear densities $\left(\rho<\rho_{\text {nuc }}\right)$, with model parameters $\mathrm{G} 1, \mathrm{G} 2, \mathrm{G} 3$, G4 and G5 corresponding to $\gamma_{1}=1.325,1.320,1.310,1.300$ and 1.280 , respectively. The name of a simulated model is then given by a combination of parameters from the three sets (e.g., A3B4G5). We define the nuclear matter density $\rho_{\text {nuc }}$ as $2.0 \times 10^{14} \mathrm{~g} \mathrm{~cm}^{-3}$.

The adiabatic index at supranuclear densities $\left(\rho \geq \rho_{\text {nuc }}\right)$ is fixed to $\gamma_{2}=2.5$, except in one model which has also been run with $\gamma_{2}=2.0$ in order to test the influence of a softer supranuclear EoS on the collapse dynamics.

In total, we have simulated the evolution of 26 models (see Table 1). In order to identify the relativistic effects on the collapse dynamics, we have simulated these models also in Newtonian gravity. Note that the Newtonian simulations are a subset of those performed by Zwerger (1995) and Zwerger \& Müller (1997).

The Einstein equations are formulated using the so-called conformally flat (CF) metric approximation (Wilson et al. 1996, conformal flatness condition - CFC) and the corresponding hydrodynamic equations are formulated as a first-order flux-conservative hyperbolic system (Banyuls et al. 1997), well-adapted to numerical schemes based on Riemann solvers. The applicability and quality of the CFC for rotational core collapse has been discussed in detail in Paper I, where we demonstrated that its usage is appropriate.

\section{Collapse dynamics}

\subsection{Collapse and waveform types}

Zwerger (1995) first identified three distinct classes of core collapse types. This classification is based on the form of the gravitational wave signal. However, as the gravitational radiation waveform is closely linked to the collapse dynamics, it also mirrors the collapse behavior. Thus, the signal types can generally be used to classify the collapse type, too.

In our relativistic simulations, each of the examined models belongs to one of Zwerger's three collapse types, which he called type I, II and III. In the following we describe a representative model from each class, discuss its characteristic properties and explain the physical effects which lead to its collapse classification. 
In general, the evolution of any supernova core collapse can be divided into three phases (see, e.g., Müller 1998):

Infall phase: this phase covers the initial collapse of the core from the onset of the gravitational instability triggered by the sudden softening of the EoS due to the reduction of the subnuclear adiabatic index. The inner part of the core, which collapses homologously $\left(v_{\mathrm{r}} \sim r\right)$, constitues the "inner core", while the "outer core" is falling supersonically. Depending on the model parameters the infall phase lasts between $30 \mathrm{~ms}$ and $100 \mathrm{~ms}$.

Bounce phase: when the EoS stiffens because repulsive nuclear forces become important at densities above nuclear density $\rho_{\text {nuc }}=2 \times 10^{14} \mathrm{~g} \mathrm{~cm}^{-3}$, or when centrifugal forces begin to dominante over gravitational attraction due to angular momentum conservation and rotational spin-up, the inner core decelerates on a timescale of about $1 \mathrm{~ms}$. Because of its large inertia and infall kinetic energy, the inner core does not come to rest immediately. The core overshoots the equilibrium configuration it will eventually approach, and bounces back, which results in the formation of a shock wave at the outer edge of the inner core. During core bounce the amplitude of the gravitational wave signal is largest.

Ring-down phase, or re-expansion phase: if centrifugal forces remain sufficiently small until nuclear density is reached in the center of the core, the bounce occurs at central densities slightly in excess of nuclear density due to the stiffening of the EoS. In this case the inner core rapidly settles down into a new equilibrium state, which we will call the "compact remnant" 2 in the following. While the shock wave propagates outwards, the inner core oscillates for about $10 \mathrm{~ms}$ with a superposition of several damped eigenmodes with frequencies of about $10^{3} \mathrm{~Hz}$ (ring-down). If the infall is moderately fast (collapse timescale longer than about $50 \mathrm{~ms}$ ), we will talk of a regular collapse model giving rise to a type I gravitational wave signal according to the nomenclature of Zwerger \& Müller (1997). In case of a very rapid infall (collapse timescale of about $30 \mathrm{~ms}$ ), the core plunges unimpeded by centrifugal forces deeply into the gravitational potential well, with its central density significantly exceeding nuclear density. In such rapid collapse models, the gravitational waveform (type III according to the nomenclature of Zwerger \& Müller 1997) is qualitatively different from that of a regular (type I) collapse model (see below).

On the other hand, if core collapse is only or predominantly stopped by centrifugal forces, the inner core experiences several distinct sequences of infall, bounce, and re-expansion separated by up to $50 \mathrm{~ms}$ before it eventually settles down into an equilibrium state. During each bounce an outward shock is generated. The multiple large scale bounces occur because the deceleration by centrifugal forces is less abrupt than that due to the stiffness of the supranuclear EoS. The latter is characterized by an adiabatic index $\gamma_{2}=2.5$ (see Sect. 2), while

\footnotetext{
${ }^{2}$ Due to neutrino cooling and other microphysical processes, which we do not consider in our models, the compact remnant later evolves into a neutron star. This may be accompanied by mass accretion due to fall-back. We have not investigated this late time evolution of the compact remnant.
}

rotation acts like a $\gamma=5 / 3$ gas according to the virial theorem (see, e.g., Tassoul 1978, Chap. 14). Multiple bounce models produce type II gravitational wave signals (Zwerger \& Müller 1997) which consist of a distinct large amplitude peak for every bounce.

If the peak central density at bounce is close to (or exceeds) nuclear matter density, and if the core rotates differentially and rapidly, a mixture of a regular and multiple collapse type model can occur. In these transition models of type I/II the core re-expands less than in a genuine multiple bounce model, but it still exhibits distinct bounces and coherent large scale oscillations.

\subsubsection{Regular collapse - type I}

As in the work of Zwerger \& Müller (1997) we choose model A1B3G3 as a representative model for a regular collapse. Figure 1 shows the time evolution of the central density $\rho_{\mathrm{c}}$ and the gravitational wave signal amplitude $A_{20}^{\mathrm{E} 2}$; the definition of the wave amplitude $A_{20}^{\mathrm{E} 2}$ and the description of the numerical wave extraction technique are given in Appendix A. The three phases of the collapse are clearly identifiable in both panels. During the infall phase $\rho_{\mathrm{c}}$ increases and exceeds nuclear matter density at $48.26 \mathrm{~ms}$. The peak value of the density is reached at the time of bounce at $t_{\mathrm{b}}=48.63 \mathrm{~ms}$ with $\rho_{\mathrm{cb}}=$ $4.23 \times 10^{14} \mathrm{~g} \mathrm{~cm}^{-3}$, which is about twice nuclear matter density. Subsequently, the core slightly re-expands and rings down to an equilibrium state with a central density of $\rho_{\mathrm{cf}} \approx 1.5 \rho_{\text {nuc }}$.

The gravitational wave amplitude (lower panel of Fig. 1) increases during the infall phase, but has a negative peak value with $\left|A_{20}^{\mathrm{E} 2}\right|_{\max }=659 \mathrm{~cm}$ at $t_{\mathrm{gw}}=48.99 \mathrm{~ms}>t_{\mathrm{b}}$. In all regular collapse models the maximum signal amplitude is negative. For some models (not in model A1B3G3) the signal amplitude exhibits a small local minimum around the time of bounce, a feature which was identified and discussed by Zwerger \& Müller (1997). The ring-down phase of the pulsating inner core is directly reflected in the wave signal which oscillates accordingly. As in the Newtonian runs of Zwerger \& Müller (1997), we find that during the ring-down phase the maxima of $A_{20}^{\mathrm{E} 2}$ are less damped than the minima, because larger accelerations are encountered at the high density extrema of the core.

\subsubsection{Multiple bounce collapse - type II}

For an adiabatic index $\gamma_{1}$ close to the initial value $\gamma_{\text {ini }}=4 / 3$ (see Sect. 2), and for rapid and highly differential rotation, centrifugal forces can halt the collapse at densities below nuclear matter density. Such subnuclear bounces can indeed be observed in some of our collapse models, e.g. model A2B4G1, for which the time evolution of the central density $\rho_{\mathrm{c}}$ and the gravitational wave signal amplitude $A_{20}^{\mathrm{E} 2}$ are shown in Fig. 2.

In both the central density evolution and the signal waveform distinct extrema are discernable. The (negative) maximum of the signal amplitude $\left|A_{20}^{\mathrm{E} 2}\right|_{\max }=548 \mathrm{~cm}$ is reached at a time $t_{\mathrm{gw}}=101.16 \mathrm{~ms}$, which is shortly after the time $t_{\mathrm{b}}=$ $99.78 \mathrm{~ms}$ when the central density reaches its first peak $\rho_{\mathrm{cb}}=$ $0.90 \times 10^{14} \mathrm{~g} \mathrm{~cm}^{-3} \approx 0.45 \rho_{\text {nuc }}$. During the subsequent bounces 


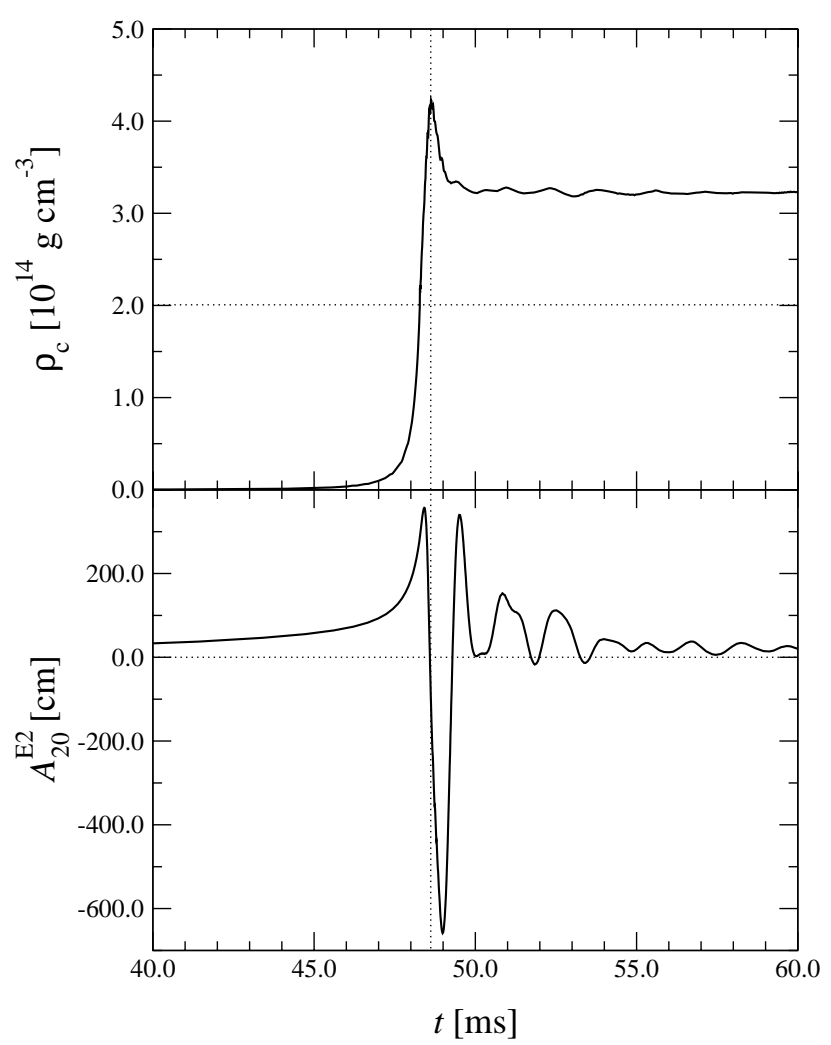

Fig. 1. Time evolution of the central density $\rho_{\mathrm{c}}$ (upper panel) and the gravitational wave signal amplitude $A_{20}^{\mathrm{E} 2}$ (lower panel) for the regular collapse model A1B3G3. The horizontal dotted line in the upper panel marks nuclear matter density $\rho_{\text {nuc }}$, and the vertical dotted line indicates the time of peak central density $t_{\mathrm{b}}$.

the peaks of the central density are significantly smaller than the peak value at first bounce. The prominent peaks in the gravitational wave signal are clearly associated with the distinct bounces visible in the density evolution.

We point out that model A1B3G1, which shows multiple bounces in Newtonian gravity (Zwerger \& Müller 1997), does not exhibit such a behavior in the relativistic simulations. This change of collapse type due to relativistic effects is discussed in Sect. 3.3.

\subsubsection{Rapid collapse - type III}

If the core collapses very rapidly due to values of $\gamma_{1}$ which are much smaller than $4 / 3$, the qualitative behavior of the density evolution is very similar to that of the regular (type I) collapse model, except that the bounce and equilibrium densities are slightly higher, and the post-bounce pulsations of the inner core are even more strongly suppressed. For example, in model A1B3G5 (Fig. 3), which is a typical rapid collapse model, the values of the peak and the final central density are $\rho_{\mathrm{cb}}=4.55 \times 10^{14} \mathrm{~g} \mathrm{~cm}^{-3} \approx 2.5 \rho_{\text {nuc }}\left(\right.$ reached at $\left.t_{\mathrm{b}}=30.20 \mathrm{~ms}\right)$, and $\rho_{\text {cf }} \approx 1.8 \rho_{\text {nuc }}$.

On the other hand, the shape of the gravitational wave signal amplitude is qualitatively different compared to type I models. This is shown in the lower panel of Fig. 3, where the time evolution of $A_{20}^{\mathrm{E} 2}$ is plotted. The maximum signal amplitude

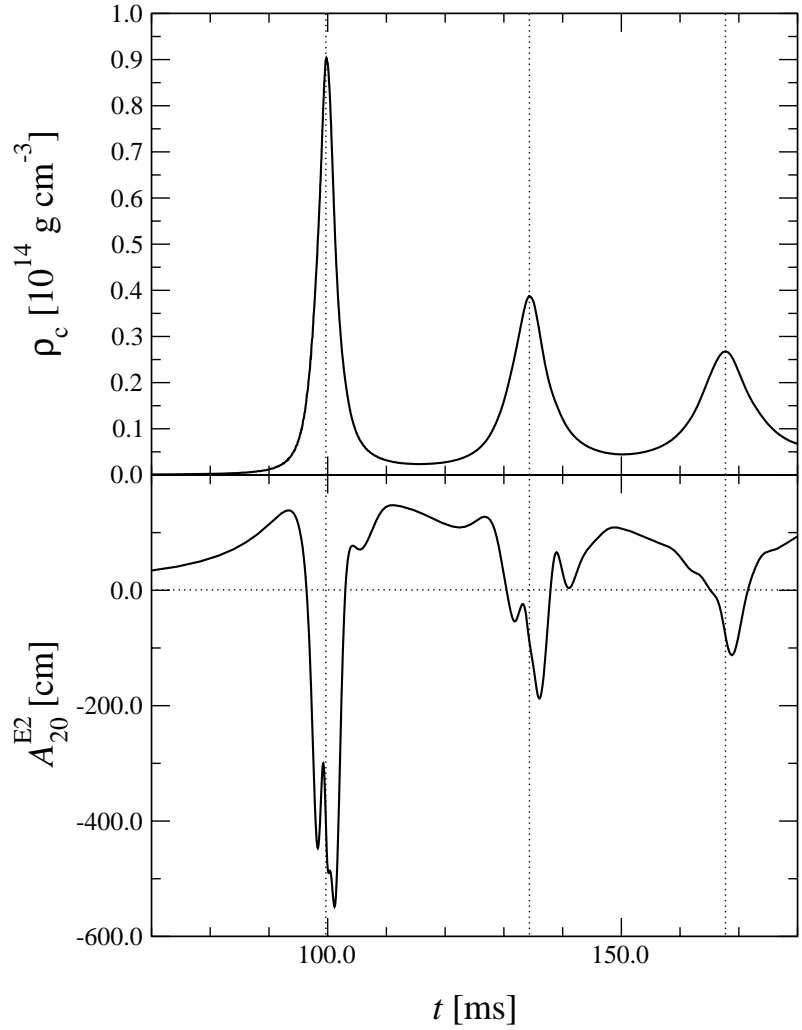

Fig. 2. Time evolution of the central density $\rho_{\mathrm{c}}$ (upper panel) and the gravitational wave signal amplitude $A_{20}^{\mathrm{E} 2}$ (lower panel) for the multiple bounce collapse model A2B4G1. The vertical dotted lines mark the times of peak central density for each bounce.

$\left|A_{20}^{\mathrm{E} 2}\right|_{\max }=124 \mathrm{~cm}$ at $t_{\mathrm{gw}}=30.03 \mathrm{~ms}$ is now assigned to the first positive peak of the waveform contrary to the signal waveform of the regular collapse models. In the case of rapid collapse, the negative peak is almost entirely suppressed and the overall amplitude of the signal is significantly smaller. There is no clear feature in the waveform allowing one to identify the time of bounce $t_{\mathrm{b}}$.

For a given initial model, the transition between type I and type III collapse models occurs gradually with decreasing values of $\gamma_{1}$. In the gravitational wave signal this is reflected by a decrease of the negative main peak and an increase of the first positive peak (Zwerger \& Müller 1997).

\subsubsection{Additional remarks}

In the above models the central density $\rho_{\mathrm{c}}$ coincides with $\rho_{\max }$, the maximum density of the model at any time. However, very rapidly and differentially rotating models, like all "A4" models, either already possess a toroidal density distribution initially, or develop one during their evolution. In such models the maximum density is attained at some off-center location, and $\rho_{\mathrm{c}}<\rho_{\max }$ holds. Nevertheless, the time evolution of $\rho_{\max }$ can be used to classify the collapse type according to the criteria discussed above.

Our results show that relativistic simulations of rotational core collapse exhibit the same qualitative collapse dynamics and allow for the same classification as Newtonian simulations 


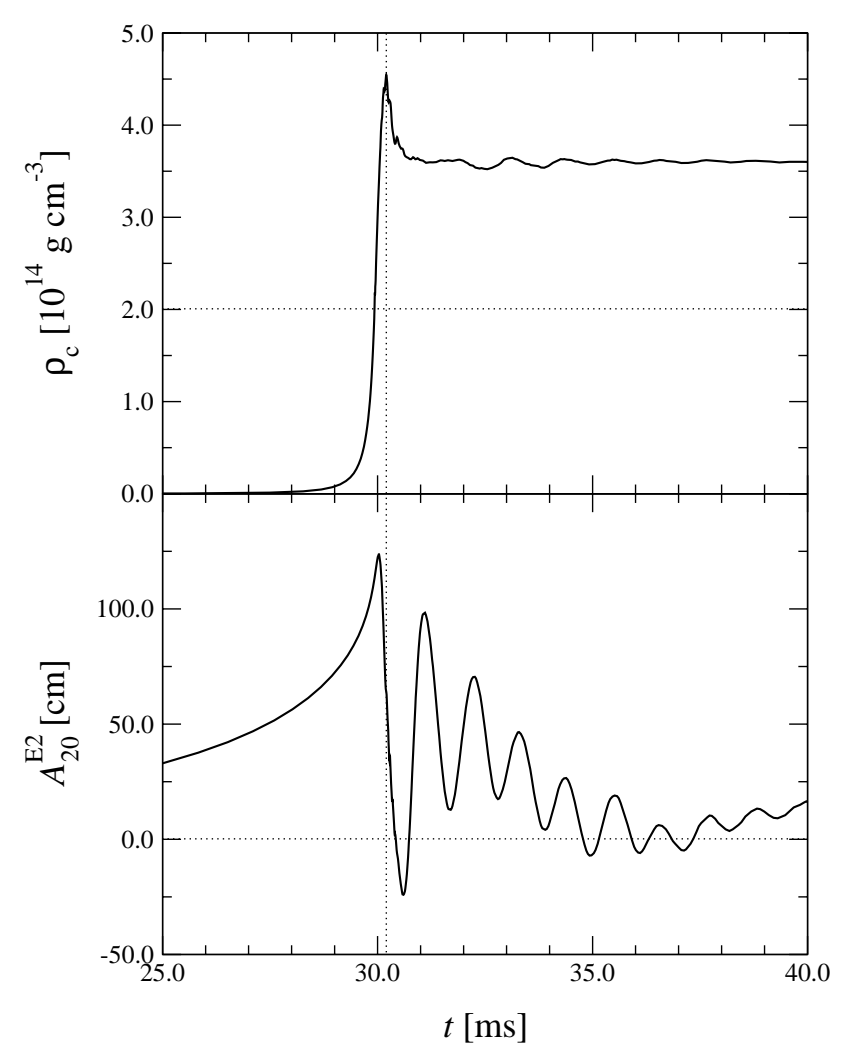

Fig. 3. Time evolution of the central density $\rho_{\mathrm{c}}$ (upper panel) and the gravitational wave signal amplitude $A_{20}^{\mathrm{E} 2}$ (lower panel) for the rapid collapse model A1B3G5. The horizontal dotted line in the upper panel marks nuclear matter density $\rho_{\text {nuc }}$, and the vertical dotted line indicates the time of peak central density $t_{\mathrm{b}}$. Note the qualitative difference in the waveform compared to the regular collapse (type I) model shown in Fig. 1.

(Zwerger 1995, 1997). However, there exist important differences in other aspects of the collapse arising from relativistic effects. These are discussed in the subsequent sections.

\subsection{Compactness of the core remnant}

For all models the central density at bounce $\rho_{\mathrm{cb}}$ (for all A4 models and model A3B5G4 the off-center maximum density $\rho_{\max }$ ) is larger in the relativistic simulation than in the corresponding Newtonian one, the relative increase reaching $\sim 700 \%$ in special cases (see Table 1 ). This also holds for the maximum density of the compact remnant after ring-down in case of instant formation of a stable equilibrium state (types I and III). This generic property is illustrated in the upper panel of Figs. 4 and 5 for the type I model A3B2G4 $4_{\text {soft }}$ (where relativistic effects are particularly large due to its soft EoS and thus high central density) and the type II model A2B4G1, respectively.

When comparing models of the same collapse type in Newtonian and relativistic gravity, we find that although all relativistic models reach higher central and/or maximum densities and, on average, larger infall and rotation velocities, only six models (see Table 1) have also larger maximum signal amplitudes than their Newtonian counterparts. The maximum

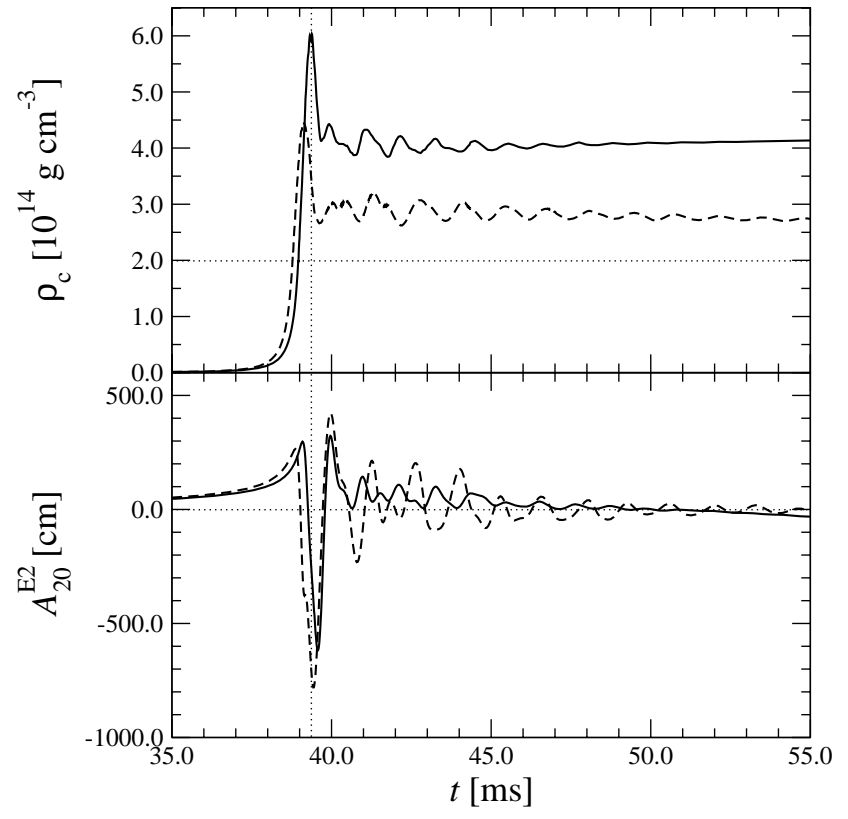

Fig. 4. Time evolution of the central density $\rho_{\mathrm{c}}$ (upper panel) and the gravitational wave signal amplitude $A_{20}^{\mathrm{E} 2}$ (lower panel) in the relativistic (solid lines) and Newtonian (dashed lines) simulation of the regular collapse model A3B2G4 $4_{\text {soft }}$. The horizontal dotted line in the upper panel marks nuclear matter density $\rho_{\text {nuc }}$. The vertical dotted line indicates the time of peak central density $t_{\mathrm{b}}$.

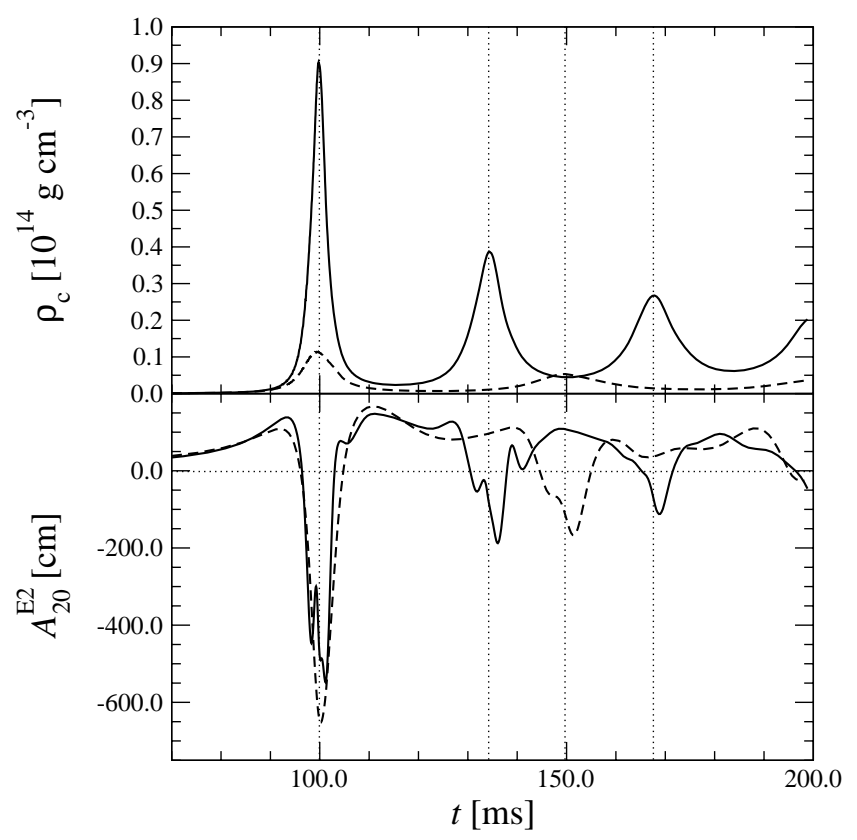

Fig. 5. Same as Fig. 4, but for the multiple bounce collapse model A2B4G1. The vertical dotted lines mark the times of peak central density $t_{\mathrm{b}}$ for each bounce.

signal amplitudes of all other relativistic models are (up to $57 \%$ ) smaller than those of the corresponding Newtonian ones (Table 1). As already discussed in Dimmelmeier et al. (2001), the reduced maximum signal strength can be explained by the fact that the amplitude, which is calculated using the quadrupole formula (see Appendix A), is determined by the bulk motion of the core rather than just by the motion of 
Table 1. Summary of important quantities for all models in relativistic $(\mathrm{R})$ and Newtonian $(\mathrm{N})$ gravity. $t_{\mathrm{b}}$ is the time of bounce, $\rho_{\max \mathrm{b}}$ is the maximum density at bounce, $\left|A_{20}^{\mathrm{E} 2}\right|_{\max }$ is the maximum gravitational wave amplitude, $v_{\max }$ is the frequency of maximum spectral energy density, $\beta_{\text {rot } \max }$ is the maximum rotation rate, $\rho_{\max }$ is the maximum density after ring-down, and Type specifies the collapse type (for a definition see text). If the maximum density is located off-center, the respective density value is marked with an asterisk. For models of type II or I/II, $\rho_{\max }$ cannot be determined.

\begin{tabular}{|c|c|c|c|c|c|c|c|c|c|c|c|}
\hline Model & $\begin{array}{c}t_{\mathrm{b}} \\
{[\mathrm{ms}]}\end{array}$ & $\begin{array}{c}\rho_{\max \mathrm{b}} \\
{\left[10^{14} \frac{\mathrm{g}}{\mathrm{cm}^{3}}\right]}\end{array}$ & $\begin{array}{c}\frac{\rho_{\operatorname{maxb}}^{\mathrm{R}}}{\rho_{\operatorname{maxb}}^{\mathrm{N}}}-1 \\
{[\%]}\end{array}$ & $\begin{array}{c}\left|A_{20}^{\mathrm{E} 2}\right|_{\max } \\
{[\mathrm{cm}]}\end{array}$ & $\begin{array}{c}\frac{\left|A_{20}^{\mathrm{E} 2}\right|_{\max }^{\mathrm{R}}}{\left|A_{20}^{\mathrm{E} 2}\right|_{\max }^{\mathrm{N}}}-1 \\
{[\%]}\end{array}$ & $\begin{array}{l}v_{\max } \\
{[\mathrm{Hz}]}\end{array}$ & $\begin{array}{c}\frac{v_{\max }^{\mathrm{R}}}{\mu_{\max }^{\mathrm{N}}}-1 \\
{[\%]}\end{array}$ & $\begin{array}{c}\beta_{\text {rot max }} \\
{[\%]}\end{array}$ & $\begin{array}{c}\frac{\beta_{\mathrm{rot} \max }^{\mathrm{R}}}{\beta_{\mathrm{rot} \max }^{\mathrm{N}}}-1 \\
{[\%]}\end{array}$ & $\begin{array}{c}\rho_{\operatorname{maxf}} \\
{\left[10^{14} \frac{\mathrm{g}}{\mathrm{cm}^{3}}\right]}\end{array}$ & Type \\
\hline $\begin{array}{l}\mathrm{A} 1 \mathrm{~B} 1 \mathrm{G} 1^{\mathrm{R}} \\
\mathrm{A} 1 \mathrm{~B} 1 \mathrm{G} 1^{\mathrm{N}}\end{array}$ & $\begin{array}{l}91.17 \\
92.23\end{array}$ & $\begin{array}{l}4.78 \\
3.22\end{array}$ & +48 & $\begin{array}{r}943 \\
1698 \\
\end{array}$ & -44 & $\begin{array}{l}623 \\
515\end{array}$ & +21 & $\begin{array}{l}5.5 \\
5.2\end{array}$ & +6 & $\begin{array}{l}3.5 \\
-\end{array}$ & $\begin{array}{r}\text { I } \\
\text { I/II }\end{array}$ \\
\hline $\begin{array}{l}\text { A1B2G1 } \\
\text { A1B2G1 }\end{array}$ & $\begin{array}{l}91.92 \\
93.10 \\
\end{array}$ & $\begin{array}{l}4.45 \\
2.89 \\
\end{array}$ & +54 & $\begin{array}{l}1803 \\
2355 \\
\end{array}$ & -23 & $\begin{array}{l}930 \\
446 \\
\end{array}$ & +109 & $\begin{array}{l}8.9 \\
8.2 \\
\end{array}$ & +9 & $\begin{array}{l}3.4 \\
- \\
\end{array}$ & $\begin{array}{r}\text { I } \\
\text { II }\end{array}$ \\
\hline $\begin{array}{l}\text { A1B3G1 }{ }^{\mathrm{R}} \\
\text { A1B3G1 }^{\mathrm{N}}\end{array}$ & $\begin{array}{l}93.25 \\
94.70\end{array}$ & $\begin{array}{l}4.07 \\
2.17 \\
\end{array}$ & +88 & $\begin{array}{l}2679 \\
1463 \\
\end{array}$ & +84 & $\begin{array}{r}1053 \\
193 \\
\end{array}$ & +446 & $\begin{array}{l}12.6 \\
10.7\end{array}$ & +18 & $\begin{array}{l}3.2 \\
-\end{array}$ & $\begin{array}{l}\text { I } \\
\text { II }\end{array}$ \\
\hline $\begin{array}{l}\text { A1B3G2 }{ }^{\mathrm{R}} \\
\text { A1B3G2 }^{\mathrm{N}}\end{array}$ & $\begin{array}{l}69.45 \\
69.87\end{array}$ & $\begin{array}{l}4.23 \\
2.83\end{array}$ & +45 & $\begin{array}{l}1718 \\
2084\end{array}$ & -18 & $\begin{array}{r}1018 \\
448\end{array}$ & +127 & $\begin{array}{l}11.9 \\
10.6\end{array}$ & +12 & $\begin{array}{l}3.2 \\
-\end{array}$ & $\begin{array}{l}\text { I } \\
\text { II }\end{array}$ \\
\hline $\begin{array}{l}\text { A1B3G3 }{ }^{\mathrm{R}} \\
\text { A1B3G3 }^{\mathrm{N}}\end{array}$ & $\begin{array}{l}48.63 \\
48.57 \\
\end{array}$ & $\begin{array}{l}4.23 \\
3.38 \\
\end{array}$ & +25 & $\begin{array}{l}659 \\
976 \\
\end{array}$ & -32 & $\begin{array}{l}702 \\
576 \\
\end{array}$ & +22 & $\begin{array}{r}10.6 \\
8.0 \\
\end{array}$ & +33 & $\begin{array}{l}3.2 \\
2.5 \\
\end{array}$ & $\begin{array}{l}\mathrm{I} \\
\mathrm{I}\end{array}$ \\
\hline $\begin{array}{l}\text { A1B3G5 } \\
\text { A1B3G5 }\end{array}$ & $\begin{array}{l}30.20 \\
29.98\end{array}$ & $\begin{array}{l}4.55 \\
4.26 \\
\end{array}$ & +7 & $\begin{array}{l}124 \\
131\end{array}$ & -5 & $\begin{array}{l}904 \\
852\end{array}$ & +6 & $\begin{array}{l}5.1 \\
3.4\end{array}$ & +50 & $\begin{array}{l}3.6 \\
3.0\end{array}$ & $\begin{array}{l}\text { III } \\
\text { III }\end{array}$ \\
\hline $\begin{array}{l}\text { A2B4G1 } \\
\text { A2B4G1 }\end{array}$ & $\begin{array}{l}99.78 \\
99.71 \\
\end{array}$ & $\begin{array}{l}0.90 \\
0.11 \\
\end{array}$ & +718 & $\begin{array}{l}548 \\
652 \\
\end{array}$ & -16 & $\begin{array}{l}86 \\
61 \\
\end{array}$ & +41 & $\begin{array}{l}17.3 \\
11.8 \\
\end{array}$ & +47 & - & $\begin{array}{l}\text { II } \\
\text { II }\end{array}$ \\
\hline $\begin{array}{l}\text { A3B1G1 } \\
\text { A3B1G1 }^{\mathrm{N}}\end{array}$ & $\begin{array}{l}91.63 \\
92.72\end{array}$ & $\begin{array}{l}4.61 \\
3.00\end{array}$ & +54 & $\begin{array}{l}1965 \\
2510\end{array}$ & -22 & $\begin{array}{r}1044 \\
482 \\
\end{array}$ & +117 & $\begin{array}{l}9.1 \\
7.5\end{array}$ & +21 & $\begin{array}{l}.5 \\
-\end{array}$ & $\begin{array}{c}\text { I } \\
\text { II }\end{array}$ \\
\hline $\begin{array}{l}\mathrm{A} 3 \mathrm{~B} 2 \mathrm{G} 1^{\mathrm{R}} \\
\mathrm{A} 3 \mathrm{~B} 2 \mathrm{G} 1^{\mathrm{N}}\end{array}$ & $\begin{array}{l}92.86 \\
94.32\end{array}$ & $\begin{array}{l}4.24 \\
2.28\end{array}$ & +86 & $\begin{array}{l}2896 \\
1711\end{array}$ & +69 & $\begin{array}{r}1133 \\
456\end{array}$ & +148 & $\begin{array}{l}13.8 \\
11.0\end{array}$ & +25 & $\begin{array}{l}3.1 \\
-\end{array}$ & $\begin{array}{l}\text { I } \\
\text { II }\end{array}$ \\
\hline $\begin{array}{l}\text { A3B2G2 }{ }^{\mathrm{R}} \\
\text { A3B2G2 }{ }^{\mathrm{N}}\end{array}$ & $\begin{array}{l}69.53 \\
69.98\end{array}$ & $\begin{array}{l}4.10 \\
2.68\end{array}$ & +53 & $\begin{array}{l}2183 \\
2407\end{array}$ & -9 & $\begin{array}{r}1128 \\
494 \\
\end{array}$ & +128 & $\begin{array}{l}13.9 \\
11.7 \\
\end{array}$ & +19 & $\begin{array}{l}3.1 \\
-\end{array}$ & $\begin{array}{l}\text { I } \\
\text { II }\end{array}$ \\
\hline $\begin{array}{l}\text { A3B2G4 } 4_{\text {soft }}^{\mathrm{R}} \\
\text { A3B2G4 }\end{array}$ & $\begin{array}{l}39.38 \\
39.14\end{array}$ & $\begin{array}{l}6.03 \\
4.45\end{array}$ & +36 & $\begin{array}{l}618 \\
781\end{array}$ & -21 & $\begin{array}{l}925 \\
755\end{array}$ & +23 & $\begin{array}{r}13.6 \\
9.5\end{array}$ & +43 & $\begin{array}{l}4.1 \\
2.7\end{array}$ & $\begin{array}{l}\text { I } \\
\text { I }\end{array}$ \\
\hline $\begin{array}{l}\text { A3B2G4 }{ }^{\mathrm{R}} \\
\text { A3B2G4 }\end{array}$ & $\begin{array}{l}39.34 \\
39.07 \\
\end{array}$ & $\begin{array}{l}4.05 \\
3.41 \\
\end{array}$ & +19 & $\begin{array}{l}517 \\
703 \\
\end{array}$ & -26 & $\begin{array}{l}838 \\
737 \\
\end{array}$ & +14 & $\begin{array}{r}13.0 \\
9.0 \\
\end{array}$ & +44 & $\begin{array}{l}3.2 \\
2.5 \\
\end{array}$ & $\begin{array}{l}\mathrm{I} \\
\mathrm{I}\end{array}$ \\
\hline $\begin{array}{l}\text { A3B3G1 }{ }^{\mathrm{R}} \\
\text { A3B3G1 }\end{array}$ & $\begin{array}{l}95.26 \\
96.57 \\
\end{array}$ & $\begin{array}{l}3.49 \\
0.47 \\
\end{array}$ & +642 & $\begin{array}{r}982 \\
1087 \\
\end{array}$ & -10 & $\begin{array}{l}827 \\
185 \\
\end{array}$ & +347 & $\begin{array}{l}20.1 \\
11.5\end{array}$ & +75 & - & $\begin{array}{r}\mathrm{I} / \mathrm{II} \\
\mathrm{II}\end{array}$ \\
\hline $\begin{array}{l}\text { A3B3G2 }{ }^{\mathrm{R}} \\
\text { A3B3G2 }\end{array}$ & $\begin{array}{l}71.28 \\
71.77\end{array}$ & $\begin{array}{l}3.58 \\
1.10\end{array}$ & +225 & $\begin{array}{l}1353 \\
1420\end{array}$ & -5 & $\begin{array}{l}846 \\
274\end{array}$ & +209 & $\begin{array}{l}20.4 \\
13.7\end{array}$ & +49 & - & $\begin{array}{r}\text { I/II } \\
\text { II }\end{array}$ \\
\hline $\begin{array}{l}\text { A3B3G33 }{ }^{\mathrm{R}} \\
\text { A3B3G3 }\end{array}$ & $\begin{array}{l}49.73 \\
49.64\end{array}$ & $\begin{array}{l}3.35 \\
2.38^{*}\end{array}$ & +41 & $\begin{array}{l}1279 \\
1480\end{array}$ & -14 & $\begin{array}{l}895 \\
363 \\
\end{array}$ & +147 & $\begin{array}{l}20.3 \\
15.7 \\
\end{array}$ & +48 & - & $\begin{array}{l}\mathrm{I} / \mathrm{II} \\
\mathrm{I} / \mathrm{II} \\
\end{array}$ \\
\hline $\begin{array}{l}\text { A3B3G5 } \\
\text { A3B3G5 }\end{array}$ & $\begin{array}{l}30.65 \\
30.36\end{array}$ & $\begin{array}{l}3.75 \\
3.45^{*}\end{array}$ & +9 & $\begin{array}{l}256 \\
263\end{array}$ & -3 & $\begin{array}{r}1074 \\
964\end{array}$ & +14 & $\begin{array}{r}13.7 \\
9.3\end{array}$ & +47 & $\begin{array}{l}3.4 \\
2.3^{*}\end{array}$ & $\begin{array}{l}\text { III } \\
\text { III }\end{array}$ \\
\hline $\begin{array}{l}\text { A3B4G } 2^{\mathrm{R}} \\
\mathrm{A} 3 \mathrm{~B} 4 \mathrm{G} 2^{\mathrm{N}}\end{array}$ & $\begin{array}{l}74.66 \\
73.99\end{array}$ & $\begin{array}{l}0.79 \\
0.16^{*}\end{array}$ & +394 & $\begin{array}{l}594 \\
894\end{array}$ & -34 & $\begin{array}{r}101 \\
85\end{array}$ & +19 & $\begin{array}{l}20.7 \\
14.7\end{array}$ & +41 & - & $\begin{array}{l}\text { II } \\
\text { II }\end{array}$ \\
\hline $\begin{array}{l}\text { A3B5G4 }{ }^{\mathrm{R}} \\
\text { A3B5G4 }\end{array}$ & $\begin{array}{l}44.46 \\
44.31 \\
\end{array}$ & $\begin{array}{l}0.30^{*} \\
0.15^{*} \\
\end{array}$ & +100 & $\begin{array}{l}487 \\
528 \\
\end{array}$ & -8 & $\begin{array}{l}103 \\
113 \\
\end{array}$ & -9 & $\begin{array}{l}28.8 \\
20.7 \\
\end{array}$ & +39 & - & $\begin{array}{l}\text { III } \\
\text { III }\end{array}$ \\
\hline $\begin{array}{l}\text { A4B1G1 } \\
\text { A4B1G1 }\end{array}$ & $\begin{array}{l}90.77 \\
91.77\end{array}$ & $\begin{array}{l}4.93^{*} \\
2.92^{*}\end{array}$ & +69 & $\begin{array}{l}2520 \\
1992\end{array}$ & +27 & $\begin{array}{r}1309 \\
580\end{array}$ & +126 & $\begin{array}{r}10.5 \\
7.4\end{array}$ & +42 & $\begin{array}{l}3.5^{*} \\
-\end{array}$ & $\begin{array}{l}\text { I } \\
\text { II }\end{array}$ \\
\hline $\begin{array}{l}\text { A4B1G2 }{ }^{\mathrm{R}} \\
\text { A4B1G2 }\end{array}$ & $\begin{array}{l}68.39 \\
68.69 \\
\end{array}$ & $\begin{array}{l}4.72^{*} \\
3.09^{*}\end{array}$ & +53 & $\begin{array}{l}2220 \\
2034 \\
\end{array}$ & +9 & $\begin{array}{r}1268 \\
946 \\
\end{array}$ & +34 & $\begin{array}{r}12.6 \\
8.5 \\
\end{array}$ & +48 & $\begin{array}{l}3.3^{*} \\
- \\
\end{array}$ & $\begin{array}{r}\text { I } \\
\text { II } \\
\end{array}$ \\
\hline $\begin{array}{l}\text { A4B2G2 }{ }^{\mathrm{R}} \\
\text { A4B2G2 }\end{array}$ & $\begin{array}{l}68.61 \\
69.02 \\
\end{array}$ & $\begin{array}{l}4.79^{*} \\
2.59^{*}\end{array}$ & +85 & \begin{tabular}{|l|}
3393 \\
2132 \\
\end{tabular} & +59 & $\begin{array}{r}1346 \\
466 \\
\end{array}$ & +189 & $\begin{array}{l}18.4 \\
12.6 \\
\end{array}$ & +46 & $\begin{array}{l}3.1^{*} \\
-\end{array}$ & $\begin{array}{r}\text { I } \\
\text { II }\end{array}$ \\
\hline $\begin{array}{l}\text { A4B2G33 } \\
\text { A4B2G3 }\end{array}$ & $\begin{array}{l}48.87 \\
48.74\end{array}$ & $\begin{array}{l}4.37^{*} \\
2.85^{*}\end{array}$ & +53 & $\begin{array}{l}2535 \\
2217\end{array}$ & +14 & $\begin{array}{r}336 \\
370 \\
\end{array}$ & +261 & $\begin{array}{l}24.0 \\
15.5 \\
\end{array}$ & +55 & $\begin{array}{l}2.9^{*} \\
- \\
\end{array}$ & $\begin{array}{r}\text { I } \\
\text { I/II }\end{array}$ \\
\hline $\begin{array}{l}\text { A4B4G4 }{ }^{\mathrm{R}} \\
\text { A4B4G4 }\end{array}$ & $\begin{array}{l}40.29 \\
39.66\end{array}$ & $\begin{array}{l}2.18^{*} \\
0.73^{*}\end{array}$ & +199 & $\begin{array}{l}1245 \\
1748\end{array}$ & -29 & $\begin{array}{l}155 \\
226\end{array}$ & -31 & $\begin{array}{l}31.8 \\
22.6\end{array}$ & +41 & - & $\begin{array}{l}\mathrm{I} / \mathrm{II} \\
\mathrm{I} / \mathrm{II}\end{array}$ \\
\hline $\begin{array}{l}\text { A4B4G5 } \\
\text { A4B4G5 }\end{array}$ & $\begin{array}{l}32.32 \\
31.91\end{array}$ & $\begin{array}{l}3.08^{*} \\
2.25^{*}\end{array}$ & +37 & $\begin{array}{l}1033 \\
2149\end{array}$ & -52 & $\begin{array}{l}227 \\
217\end{array}$ & +5 & $\begin{array}{l}43.2 \\
28.0\end{array}$ & +54 & - & $\begin{array}{l}\mathrm{I} / \mathrm{II} \\
\mathrm{I} / \mathrm{II}\end{array}$ \\
\hline $\begin{array}{l}\text { A4B5G4 }{ }^{\mathrm{R}} \\
\text { A4B5G4 }^{\mathrm{N}}\end{array}$ & $\begin{array}{l}38.26 \\
37.29\end{array}$ & $\begin{array}{l}0.98^{*} \\
0.38^{*}\end{array}$ & +158 & $\begin{array}{l}1651 \\
2965\end{array}$ & -44 & $\begin{array}{l}90 \\
76\end{array}$ & +18 & $\begin{array}{l}38.4 \\
27.9\end{array}$ & +38 & - & $\begin{array}{l}\mathrm{I} / \mathrm{II} \\
\mathrm{I} / \mathrm{II}\end{array}$ \\
\hline $\begin{array}{l}\text { A4B5G5 } \\
\text { A4B5G5N }\end{array}$ & $\begin{array}{l}31.39 \\
30.82\end{array}$ & $\begin{array}{l}3.24^{*} \\
2.65^{*}\end{array}$ & +22 & $\begin{array}{l}2356 \\
5444\end{array}$ & -57 & $\begin{array}{l}106 \\
159\end{array}$ & -33 & $\begin{array}{l}48.6 \\
38.1\end{array}$ & +28 & - & $\begin{array}{l}\text { I/II } \\
\text { I/II }\end{array}$ \\
\hline
\end{tabular}




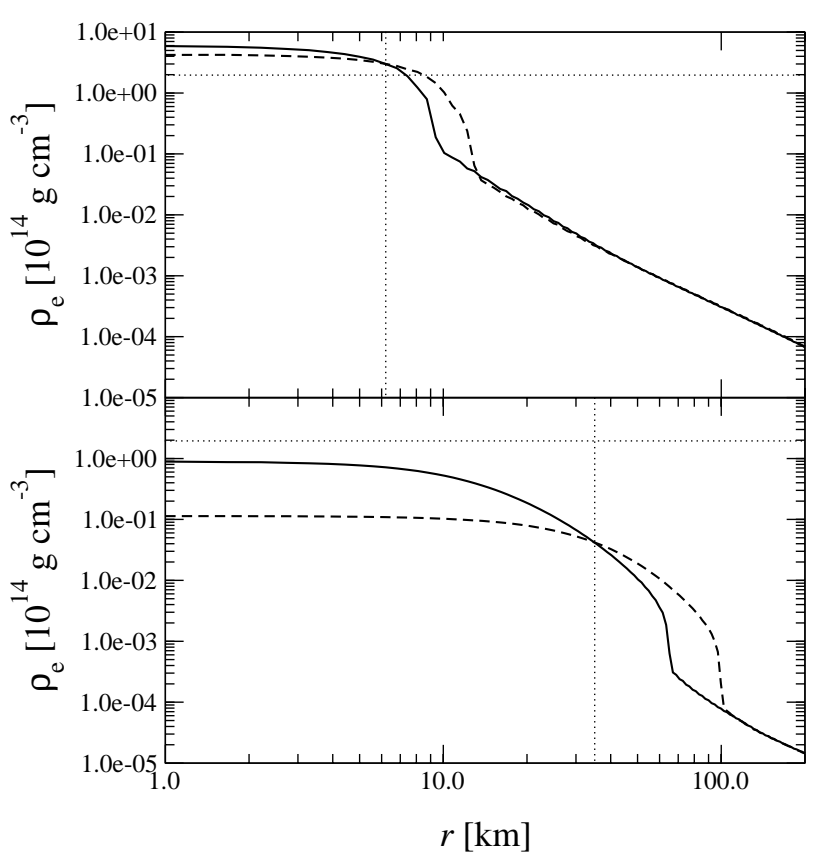

Fig. 6. Radial density profiles $\rho_{\mathrm{e}}$ along the equator near bounce time for model A3B2G4 $4_{\text {soft }}$ (upper panel) and model A2B4G1 (lower panel). In both models, the density of the relativistic simulation (solid line) is larger than that of the Newtonian simulation (dashed line) in the central region. At $r \approx 6 \mathrm{~km}$ (upper panel) and $r \approx 35 \mathrm{~km}$ (lower panel) the density of the relativistic model drops below that of the Newtonian one. The radii of the density crossing are indicated by the vertical dotted lines, and the horizontal dotted lines mark nuclear matter density $\rho_{\text {nuc }}$.

the densest mass shells, strictly speaking by the second time derivative of the quadrupole moment. Therefore, a core which is more condensed in the center can give rise to a smaller gravitational wave signal than a core which is less centrally condensed, but which is denser and moves faster in its outer regions. This is reflected by the weight factor $r^{2}$ in the integrand of the quadrupole formula (A.4). Due to this factor matter at moderately large $r$ contributes significantly to the gravitational wave signal amplitude, while at very large radii (outside the inner core) the decrease in density more than compensates the increase of the weight factor.

The upper panel of Fig. 6 shows the equatorial density profiles in the inner core of model $\mathrm{A} 3 \mathrm{~B} 2 \mathrm{G} 4_{\text {soft }}$ for both the relativistic (solid line) and the Newtonian (dashed line) simulation at bounce. As already seen in the central density evolution (Fig. 4), the simulation in relativistic gravity yields up to $36 \%$ larger densities in the central regions. However, at radii $r \gtrsim 5 \mathrm{~km}$ the density in the Newtonian model exceeds that of the relativistic one. This "density crossing", which takes place at different radii for different polar angles $\theta$, can be observed in all the models we have investigated, at all times $t>t_{\mathrm{b}}$ and at all polar angles. The surface of density crossing is located inside the compact remnant for type I and type III models, and in the case of type II multiple bounce models in the coherently re-expanding and contracting inner core, which has not yet settled into a compact remnant at the end of the simulation. The effect is persistent in time as demonstrated in Fig. 7, which

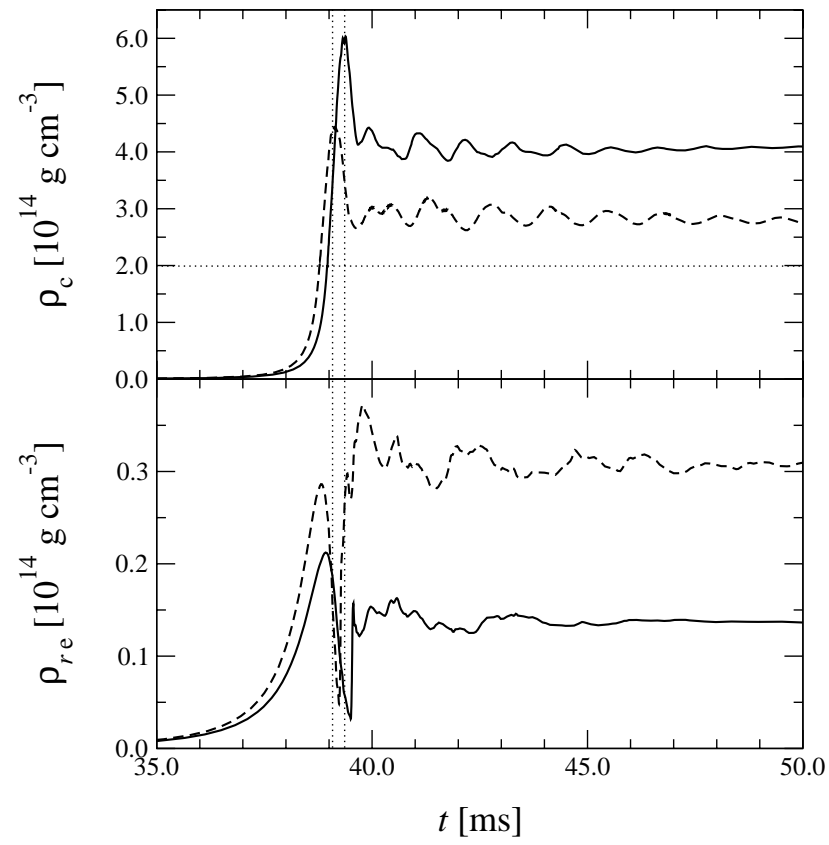

Fig. 7. Time evolution of the density at different radii in the relativistic (solid lines) and Newtonian (dashed lines) simulation of model A3B2G4 $4_{\text {soft }}$. Whereas the central density (upper panel) is larger in relativistic gravity, the (equatorial) density of the Newtonian model exceeds that of the relativistic model at a radius of $r=12.9 \mathrm{~km}$ (lower panel). The vertical dotted lines indicate the time of bounce $t_{\mathrm{b}}$, and the horizontal dotted line in the upper plot indicates nuclear matter density $\rho_{\text {nuc }}$. Note the different vertical scale of the two plots.

shows the time evolution of the density for model A3B2G4 $4_{\text {soft }}$ at two different radial locations in the equatorial plane. After bounce, the central density $\rho_{\mathrm{c}}$ of the relativistic configuration is always larger than the corresponding Newtonian one (upper panel). However, at larger radii, but still inside the compact remnant (lower panel), the density of the relativistic model is smaller by up to $50 \%$.

The analysis of the multiple bounce model A2B4G1, which yields a smaller signal amplitude in relativistic gravity (see lower panel of Fig. 5), leads to a similar result as the regular bounce model A3B2G4 $4_{\text {soft }}$. In the relativistic case, the central density at bounce is more than 8 times larger than in the Newtonian one. As in model A3B2G4 $4_{\text {soft }}$, the equatorial density profiles cross (lower panel of Fig. 6), but now the crossing occurs at a much larger radius $r \approx 35 \mathrm{~km}$. Hence, the region where the relativistic model is denser is considerably more extended. Inside the crossing radius, the cores of both the relativistic and the Newtonian model oscillate coherently. The density of the relativistic model is considerably higher in this region, and the matter experiences stronger accelerations, which causes narrower density peaks and shorter time intervals between two consecutive bounces (Fig. 5).

The effects of the reduced density in the outer regions of the inner core in relativistic gravity on the gravitational wave signal can be inferred from Fig. 8, which shows the equatorial density profile weighted by a factor $r^{2}$. Inside the crossing radius $r \approx 6 \mathrm{~km}$, the profiles of the weighted den-

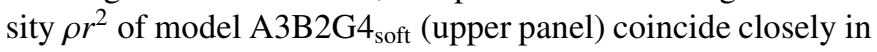



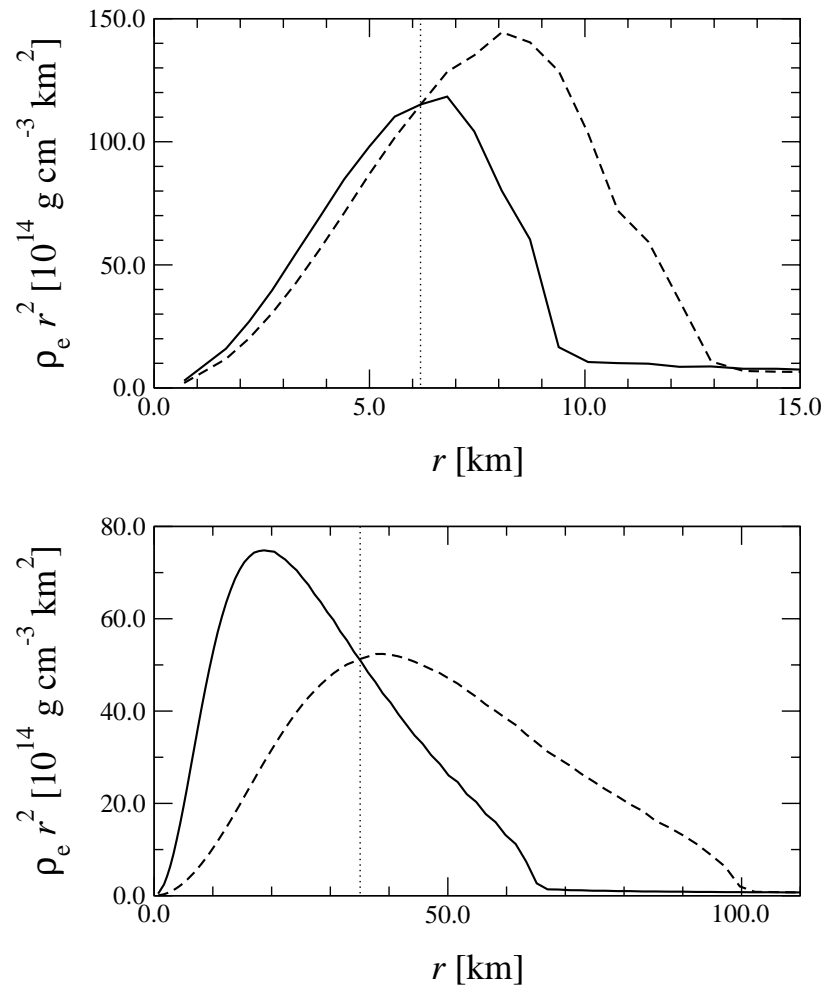

Fig. 8. Weighted radial density profile $\rho_{\mathrm{e}} r^{2}$ along the equator (solid lines denote relativistic models; dashed lines denote Newtonian models) close to the time of core bounce for model A3B2G4 $4_{\text {soft }}$ (upper panel; $t=39.25 \mathrm{~ms}$ ) and model A2B4G1 (lower panel; $t=99.75 \mathrm{~ms}$ ), respectively. The crossing radii are indicated by the vertical dotted lines.

both relativistic and Newtonian gravity. On the other hand, for $6 \mathrm{~km} \lesssim r \lesssim 13 \mathrm{~km}$ the weighted density $\rho r^{2}$ of the Newtonian model is significantly larger than that of the relativistic model. Consequently, as the density crossing occurs at all polar angles, the resulting quadrupole moment (Eq. (A.4)) is larger, too. In order to see whether the enhanced quadrupole moment also gives rise to a stronger gravitational wave signal, one has to consider the second time derivative of the quadrupole moment (see Eq. (A.4)), which results from the acceleration of the matter distribution in the core. We find that despite the somewhat lower average acceleration in Newtonian gravity which is reflected in the $18 \%$ lower frequency of the gravitational wave signal, the large quadrupole moment of the more extended density distribution results in a maximum signal amplitude $\left|A_{20}^{\mathrm{E} 2}\right|_{\max }$, which is $26 \%$ larger than that of the centrally denser compact remnant in relativistic gravity (see lower panel of Fig. 4 and Table 1).

For the multiple bounce model A2B4G1, the situation is similar (lower panel). In relativistic gravity the weighted density $\rho r^{2}$ is enhanced in the central parts of the inner core, and reduced in the outer parts compared to the Newtonian profile. Contrary to model A3B2G4 $4_{\text {soft }}$, the relativistic density increase close to the center has a strong effect on the weighted density profile inside the crossing radius $r \approx 35 \mathrm{~km}$. Nevertheless, and despite the higher accelerations (i.e., larger second time derivatives) of the coherently oscillating inner core in relativistic gravity, the smaller densities at larger radii again yield a smaller gravitational wave signal (see lower panel of Fig. 5). However, in relativistic gravity the increase of both the central density (718\% vs. $36 \%)$ and the frequency (41\% vs. $23 \%)$ are larger in model A3B2G4 $4_{\text {soft }}$ compared to model A2B4G1. Thus, the maximum signal amplitude $\left|A_{20}^{\mathrm{E} 2}\right|_{\max }$ of the former model is only reduced by $16 \%$ instead of $21 \%$ in model A2B $4 \mathrm{G} 1$ when changing from Newtonian to relativistic gravity (see Table 1).

The above considerations also explain the much weaker gravitational wave signal of model A1B1G1 in case of relativistic gravity (see upper panel in Fig. 9). In general relativity the central parts of the inner core collapse to almost $2.5 \rho_{\text {nuc }}$, and rapidly settle down to an equilibrium state with constant density after a single bounce (middle panel). Since the matter is more concentrated towards the center, the density is relatively small in the outer parts of the core (lower panel). The fluid motion in the outer regions of the core near $r=13.0 \mathrm{~km}$, where the shock forms, is not in phase with the fluid motion in the inner regions (see two lower panels in Fig. 9). In Newtonian gravity the inner core is much less compact. It has a lower central density (middle panel), but a much higher density at $r=13.0 \mathrm{~km}$ (lower panel). In this case the entire inner core oscillates coherently with large amplitude motions, which give rise to a large time variation of the quadrupole moment and thus a large gravitational wave signal, the maximum amplitude being almost twice as large as the corresponding one in relativistic gravity (see Table 1).

\subsection{Change of the collapse dynamics}

In the comprehensive study of Newtonian rotational core collapse carried out by Zwerger \& Müller (1997), models with multiple bounces were observed quite often. Relativistic gravity can have a qualitative impact on the dynamics of these models. If the density increase due to the deeper relativistic potential is sufficiently large, a collapse which is stopped by centrifugal forces at subnuclear densities (and thus undergoes multiple bounces) in a Newtonian simulation, becomes a regular, single bounce collapse in relativistic gravity.

Only two of the 26 models of our sample, A2B4G1 and A3B4G2, show unambiguous multiple bounces in relativistic gravity. These two models share some common features. In the relativistic simulations their peak central densities associated with the individual bounces are much larger (up to a factor 7) compared to those of the Newtonian runs (Table 1). Consequently, the time elapsed between subsequent bounces decreases by a factor of up to 4 . These type II models also show the effect of density crossing (see Sect. 3.2). As a result, despite the increase of the central density and the decrease of the oscillation timescale (giving rise to a larger average acceleration of the inner parts of the core), the maximum gravitational wave amplitude is lower for the relativistic models.

When the central densities become very large, relativistic gravity can even change the collapse dynamics qualitatively, i.e. the collapse type is altered with respect to the Newtonian case (see Table 1). We have observed mostly transitions from a Newtonian type II (multiple bounce collapse) to a relativistic type I (regular collapse) case, and less frequently transitions 


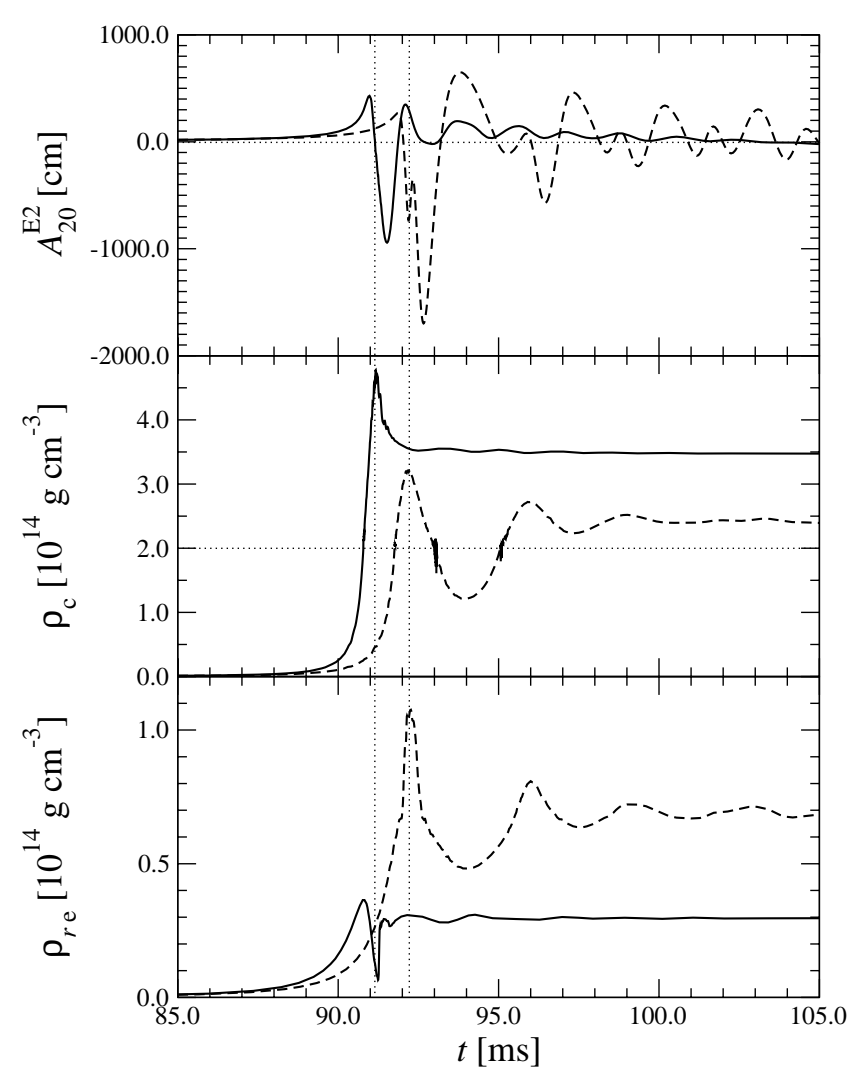

Fig. 9. Effects of a coherent motion of the inner core on the gravitational wave signal in the relativistic (solid lines) and Newtonian (dashed lines) simulation of model A1B1G1. In the upper panel the evolution of the gravitational wave amplitude $A_{20}^{\mathrm{E} 2}$ is plotted. The middle and lower panels show the evolution of the central density $\rho_{\mathrm{c}}$ and of the equatorial density $\rho_{\text {re }}$ evaluated at a radius $r=13.0 \mathrm{~km}$, respectively. Contrary to the relativistic simulation, the inner core shows large amplitude oscillations in Newtonian gravity, which causes a much larger gravitational wave signal. The vertical dotted lines indicate the time of bounce $t_{\mathrm{b}}$, and the horizontal dotted line in the middle panel marks nuclear matter density $\rho_{\text {nuc }}$.

from or to type I/II. A typical example is model A1B3G1 (Fig. 10). The change of type is clearly visible both in the evolution of the central density (upper panel) and of the gravitational wave signal (lower panel).

Centrifugal forces cause most of the type II multiple bounce models of Zwerger \& Müller (1997) to bounce at densities below $\rho_{\text {nuc }}$. Only a few models collapse to nuclear matter density, the largest maximum density at bounce being $\rho_{\max \mathrm{b}}=$ $3.09 \times 10^{14} \mathrm{~g} \mathrm{~cm}^{-3}$ in the case of model A4B1G2. Accordingly, in the two relativistic type II models the maximum bounce density does not exceed this threshold. Whenever the deeper relativistic gravitational potential drives the maximum density in the core beyond the threshold of $\rho_{\max \mathrm{b}} \gtrsim 3 \times 10^{14} \mathrm{~g} \mathrm{~cm}^{-3}$, a type transition occurs (see Table 1).

A collapse type transition does not only alter the signal waveform qualitatively, but also has important consequences for the maximum gravitational wave signal amplitude, as e.g. in the case of model A1B1G1 (Fig. 9). In this Newtonian type I/II model the concurrence of both high densities and a coherent

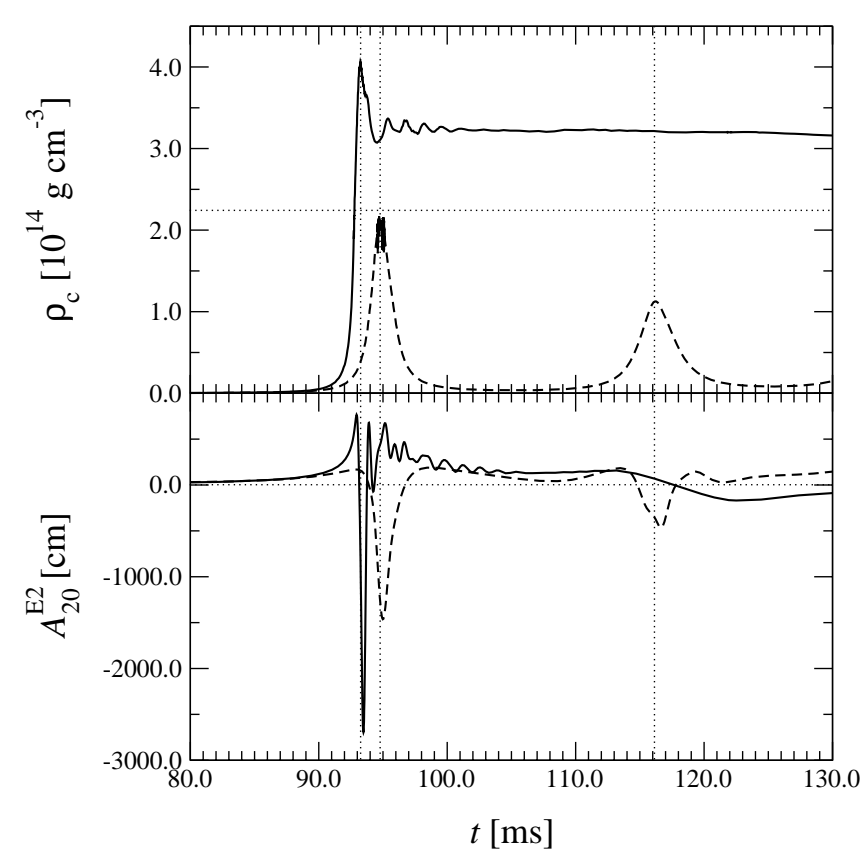

Fig. 10. Evolution of the central density $\rho_{\mathrm{c}}$ (upper panel) and the gravitational wave signal amplitude $A_{20}^{\mathrm{E} 2}$ (lower panel) in the relativistic (solid lines) and Newtonian (dashed lines) simulation of model A1B3G1. The vertical dotted lines mark the times of peak central density for each bounce, and the horizontal dotted line in the density plot marks nuclear matter density $\rho_{\text {nuc }}$.

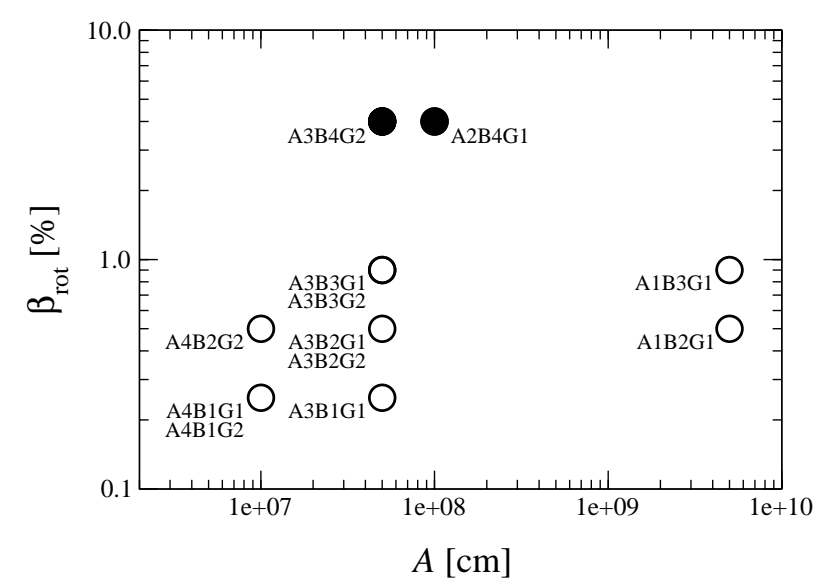

Fig. 11. Parameter space spanned by the rotation law parameter $A$ and the rotation rate $\beta_{\text {rot }}$ for multiple bounce collapse models in relativistic and Newtonian simulations. Filled circles mark models which are of multiple bounce type both in relativistic and Newtonian gravity, and empty circles mark those models which show multiple bounces in Newtonian gravity only.

motion of the inner core yields a large gravitational wave amplitude of $\sim 1700 \mathrm{~cm}$. As the stronger relativistic gravitational potential leads to the almost immediate formation of a new equilibrium state, efficiently suppressing coherent large amplitude motions of the inner core, the maximum signal amplitude is only $943 \mathrm{~cm}$, which is $44 \%$ less than in the Newtonian case.

In Newtonian gravity and for an adiabatic index $\gamma_{1}=1.325$ or $\gamma_{1}=1.320$ (i.e. close to the initial value $\gamma_{\text {ini }}=4 / 3$ ) even models with rather small rotation rates $\beta_{\text {rot ini }}$ and moderately 


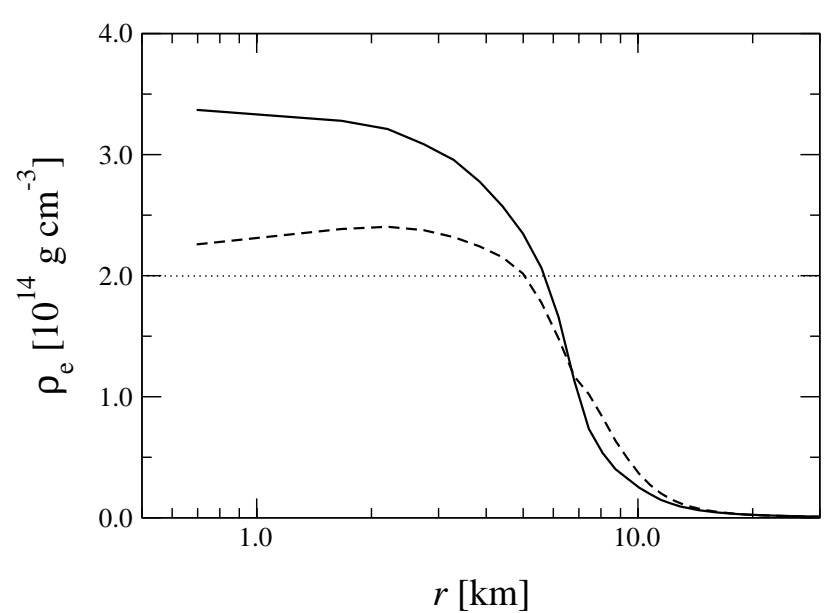

Fig. 12. Equatorial density profiles of the moderately rapidly rotating model A3B3G5 in case of relativistic (solid line) and Newtonian (dashed line) gravity, depicted after ring-down. In relativistic gravity the core has an oblate density structure. The less deep Newtonian gravitational potential leads to the development of a toroidal density configuration. The horizontal dotted line marks nuclear matter density $\rho_{\text {nuc }}$.

differential rotation (i.e. larger values of $A$ ) show multiple bounces. Our simulations demonstrate that relativistic gravity drastically narrows the region in the parameter space of $\beta_{\text {rot ini }}$ and $A$ where multiple bounce models are possible. Figure 11 shows that only models with high rotation rates and moderate differential rotation undergo an unambiguous type II multiple bounce collapse.

\subsection{Rapidly and highly differentially rotating core collapse}

When rapidly (large $\beta_{\text {rot ini }}$ ) and strongly differentially (small $A$ ) rotating initial models collapse on a short timescale due to a low adiabatic exponent $\gamma_{1}$, angular momentum conservation results in a considerable spin-up of the core. Consequently, the increasing centrifugal forces stop the collapse before or slightly above nuclear matter density, as in type II multiple bounce models. However, the maximum density is reached off-center, the topology of the density distribution in the core being toruslike. Some initial models (e.g. A4B4 and A4B5) already exhibit a toroidal structure. During collapse the toroidal character of such a model is significantly enhanced, and the maximum density $\rho_{\max }$ can exceed the central density $\rho_{\mathrm{c}}$ by up to two orders of magnitude. Other rapidly and highly differentially rotating initial models, like A3B5, A4B1 and A4B2, in general do not change their initial oblate density stratification during the evolution. However, for particular values of the parameter $\gamma_{1}$, they also develop a toroidal topology during the infall phase.

Due to their deeper gravitational potential, relativistic configurations tend to be more compact compared to the corresponding Newtonian ones (see Sect. 3.2). This also holds for relativistic models with a toroidal density stratification, i.e. for models rotating rapidly and strongly differentially. These models have off-center density maxima which exceed those of their Newtonian counterparts (see Table 1). In some borderline models which rotate moderately fast and not too differentially, the core develops a toroidal density stratification only in Newtonian gravity and possesses an oblate spheroidal density stratification in relativistic gravity. A prototype of such a case is model A3B3G5 (see Fig. 12).

In both relativistic and Newtonian simulations of rapidly and highly differentially rotating cores, the gravitational wave amplitudes are generally large, because of the large quadrupole moments of toroidal configurations (see Table 1). If the rotation rate is modest (models A4B1 and A4B2), the density in the torus at bounce can reach high supranuclear densities in relativistic gravity. The characteristic frequencies of the gravitational wave signal of these toroidal models become particularly high, as they change to regular collapse type models in relativistic gravity. The combination of a high density torus and large accelerations (reflected by the higher frequencies) yields very large wave amplitudes up to almost $3400 \mathrm{~cm}$, which is significantly larger than in Newtonian gravity. However, in the case of the extremely rapidly rotating models A4B4 and A4B5, centrifugal forces surmount relativistic gravity. The collapse type does not change, and the bounce is partially due to centrifugal forces (type I/II collapse). Consequently, the densities at bounce are less extreme and the average accelerations are smaller than in less rapidly rotating toroidal models. Therefore, the maximum signal amplitudes $\left|A_{20}^{\mathrm{E} 2}\right|_{\max }$ of models A4B4 and A4B5 are smaller than in the corresponding Newtonian models.

Compared to models with a moderate amount of rotation, the propagation of the shock wave is drastically different in a rapidly and differentially rotating model, as e.g. in model A4B5G5 (Fig. 13). In this model the density maximum is located off-center already during collapse (left panel). After core bounce the inner core settles down to an approximate equilibrium state with a distinctive toroidal density stratification surrounded by an equatorial disk from which matter is accreted onto the torus (middle panel). In this accretion disk the shock encounters relatively dense matter with large infall velocities between $0.1 c$ and $0.15 c$, and thus it propagates outward comparatively slowly along this direction. On the other hand, the region close to the rotation axis has been emptied of matter during the collapse, the average density being orders of magnitude lower than close to the equatorial plane. Therefore, here the shock propagates out very rapidly (right panel). As a result, the flow velocity in the meridional plane exhibits a jet-like structure, with matter being ejected from the inner core predominantly along the rotation axis.

\subsection{Evolution of the rotation rate}

The influence of relativistic gravity on the evolution of the rotation rate during core collapse is demonstrated in Fig. 14, which shows the radial profiles of $v_{\varphi}=\sqrt{v_{3} v^{3}}$ at the time of core bounce for several representative models. Both the maximum and average rotation velocities are higher in the relativistic models, and the maximum of $v_{\varphi}$ is shifted to smaller radii. Note that this maximum is located near the edge of the compact remnant. The differences are most pronounced for model A1B3G1 

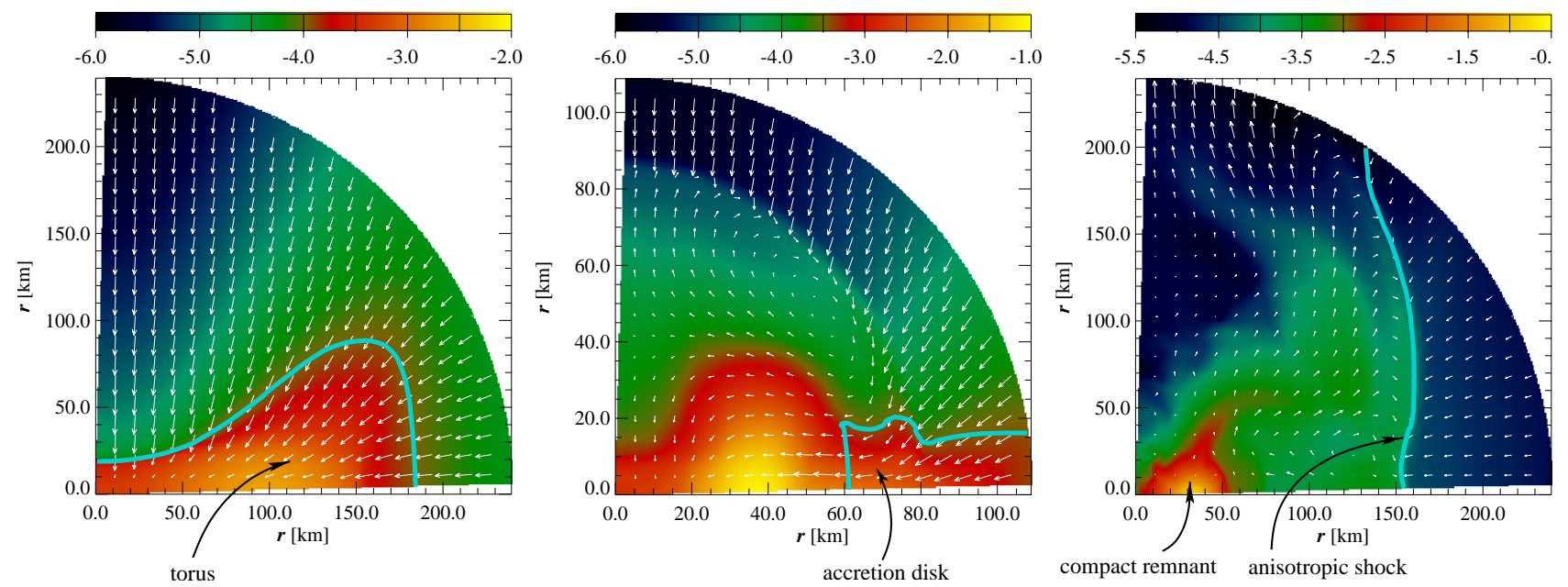

Fig. 13. Formation of a torus and shock-propagation in the very rapidly and highly differentially rotating model A4B5G5. The three snapshots show color coded contour plots of the logarithm of the density, $\log \rho$ (scaled to nuclear matter density), together with the meridional flow field during the infall phase at $t=25.0 \mathrm{~ms}$ (left panel), shortly before the centrifugal bounce at $t=31.2 \mathrm{~ms}$ (middle panel), and at $t=35.0 \mathrm{~ms}$ (right panel) when the torus is surrounded by large scale flow vortices. The length of the velocity vectors is scaled to the maximum value in the plotted region. Note that the size of the displayed region and the color coding (which is given above each panel) vary from plot to plot.

(lower panel), which changes from multiple bounce collapse to regular collapse due to relativistic effects (see Sect. 3.3). Model A1B3G1 also shows the largest rotation velocities of all our models, $v_{\varphi}$ approaching $0.2 c$ at $r \approx 15 \mathrm{~km}$ during core bounce. This corresponds to a rotation period of $T \sim 1.6 \mathrm{~ms}$ at the edge of the inner core. When collapsing from the same initial model (A1B3), but imposing a larger initial reduction of the adiabatic index to $\gamma_{1}=1.280$ (i.e. model A1B3G5; upper panel in Fig. 14), the spin-up is less and $v_{\varphi} \lesssim 0.08 c$. This result can be explained as follows: in model A1B3G1 the reduced adiabatic index $\gamma_{1}=1.325$ is very close to $4 / 3$, i.e. a large fraction of the core collapses nearly homologously (Goldreich \& Weber 1980). However, this does not hold for model A1B3G5, where the outer parts of the core fall much slower than in model A1B3G1, and hence also fall less far in radius until the time of bounce. Consequently, the spin-up of mass zones located initially at the same radial position in both models is less in model A1B3G5 than in model A1B3G1 except for the very center of the core.

Assuming angular momentum conservation for the core, the rotation rate $\beta_{\text {rot }}$ scales like the inverse of the radius of the core (see, e.g., Shapiro \& Teukolsky 1983),

$\beta_{\text {rot }}=\frac{E_{\text {rot }}}{\left|E_{\mathrm{pot}}\right|} \sim \frac{J^{2}}{M^{3} R} \propto \frac{1}{R}$

where $E_{\mathrm{rot}}, E_{\mathrm{pot}}, M, R$ and $J$ are the rotational energy, potential energy, mass, radius and angular momentum of the core, respectively.

Knowing the behavior of the evolution of $\beta_{\text {rot }}$ during core collapse is important for several reasons. Firstly, the rotation state of the inner core after bounce determines the rotation rate and profile of the compact remnant provided no significant fallback of matter occurs later in the evolution. Pulsar observations show that neutron stars can spin with very high rotation periods of the order of milliseconds (see Lorimer 2001 and references therein). However, such millisecond pulsars are believed
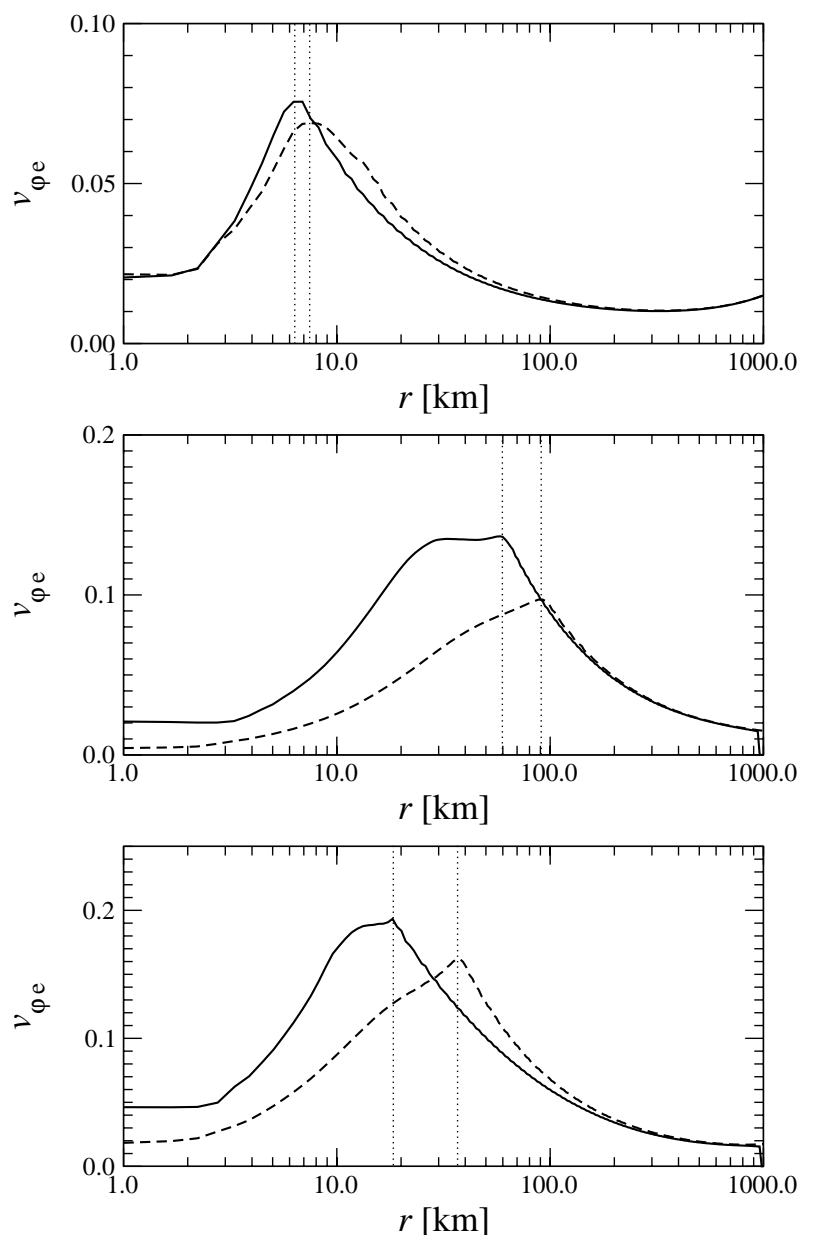

Fig. 14. Radial profiles of the rotation velocity $v_{\varphi}$ in the equatorial plane plotted shortly after core bounce obtained in the relativistic (solid lines) and Newtonian (dashed lines) simulation of models A1B3G5 (upper panel), A2B4G1 (middle panel) and A1B3G1 (lower panel). The vertical dotted lines mark the radius of maximum rotation velocity. 

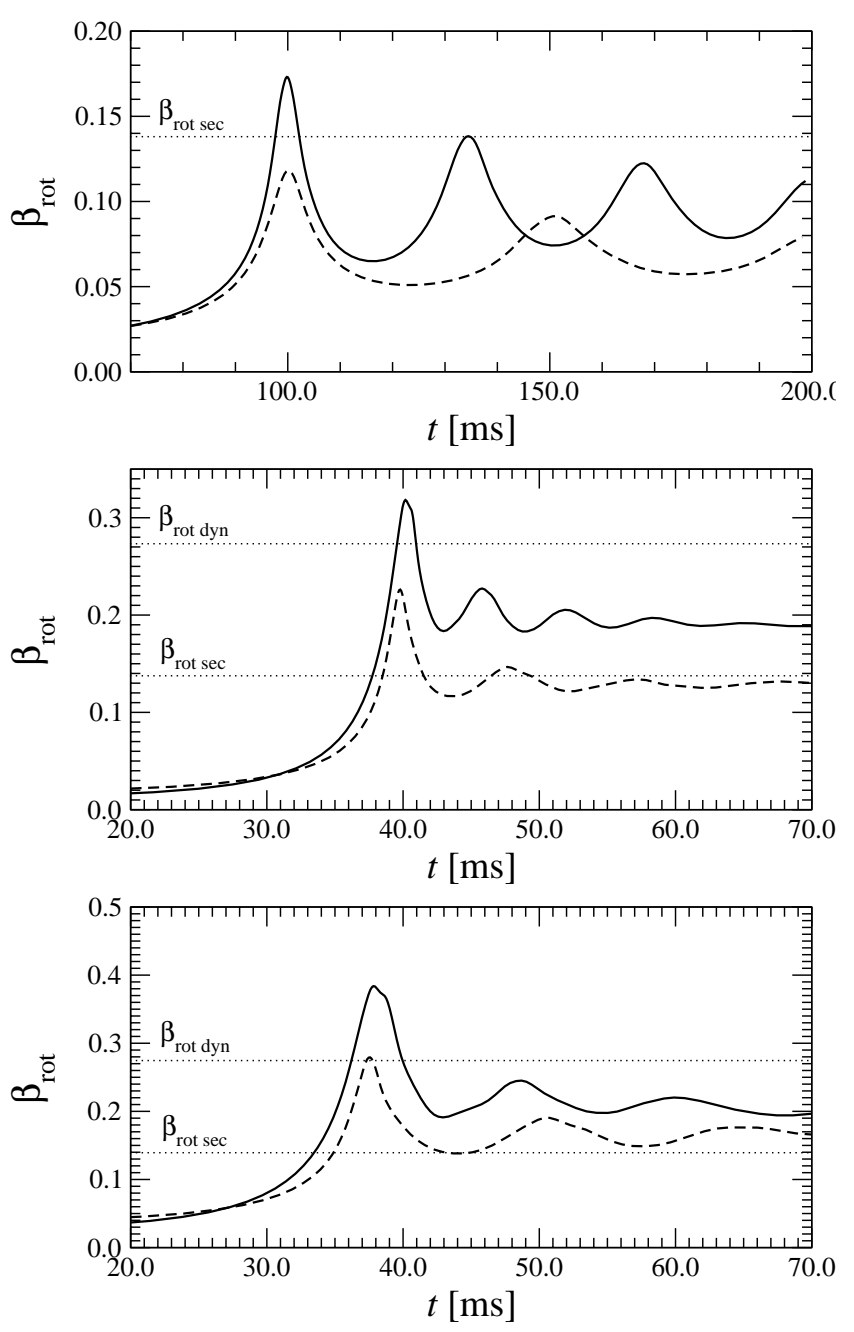

Fig. 15. Evolution of the rotation rate $\beta_{\text {rot }}$ in the relativistic (solid lines) and Newtonian (dashed lines) simulations of models A2B4G1 (upper panel), A4B4G4 (middle panel) and A4B5G4 (lower panel). The horizontal lines mark the critical rotation rates above which MacLaurin spheroids are secularly $\left(\beta_{\text {rot sec }}\right)$ and dynamically $\left(\beta_{\text {rot dyn }}\right)$ unstable against triaxial perturbations in Newtonian gravity.

to be born not as fast rotators, but are thought to be old objects which are spun up via mass transfer from a companion star much later in their evolution. Observations of pulsars in young supernova remnants (see, e.g. Kaspi 2000) provide only upper limits on the rotational state of newborn neutron stars, because significant angular momentum losses may have occurred since their formation. Presently, the fastest known young pulsar is the $16 \mathrm{~ms}$ X-ray pulsar in the Crab-like supernova remnant N157B (Marshall et al. 1998). Secondly, at sufficiently high rotation rates the core will become unstable against triaxial perturbations due to various mechanisms (see Stergioulas 1998 and references therein). Such nonaxisymmetric rotating configurations are an additional source of gravitational radiation.

For uniformly rotating, constant density MacLaurin spheroids in Newtonian gravity, the threshold values for the development of triaxial instabilities on secular and dynamic timescales are $\beta_{\text {rot sec }} \approx 13.8 \%$ and $\beta_{\text {rot dyn }} \approx 27.4 \%$, respectively (see, e.g., Tassoul 1978).
The secular instability, which is driven for example by gravitational radiation or viscosity, has been analyzed in compressible stars, both uniformly and differentially rotating, within linear perturbation theory in full general relativity by Bonazzola et al. (1996, 1998), Stergioulas \& Friedman (1998), and Yoshida \& Eriguchi (1999). The analysis shows that $\beta_{\text {rot sec }}$ depends on the compactness of the star, on the rotation law, and on the dissipative mechanism. Whereas $\beta_{\text {rot sec }}<0.14$ for the gravitational radiation-driven instability (for extremely compact or strongly differentially rotating stars $\beta_{\text {rot sec }}<0.1$ ), secular instabilities driven by viscosity occur for larger rotation rates, i.e. for values $\beta_{\text {rot sec }}>0.14$, when the configurations become more compact.

The dynamic instability, on the other hand, is driven by hydrodynamics and gravity. The onset of the dynamic barmode instability has been investigated in full general relativity only recently (Shibata et al. 2000; Saijo et al. 2001), because it requires fully nonlinear multidimensional simulations (for Newtonian investigations see, e.g., New et al. (2000), and references therein). The simulations show that in relativistic gravity the critical rotation rates for the onset of the dynamic bar-mode instability are only slightly smaller $\left(\beta_{\text {rot dyn }} \sim 0.24-0.25\right)$ than in the Newtonian case, and depend only very weakly on the degree of differential rotation (within the moderate range surveyed by the investigations).

What do these general considerations concerning triaxial instabilities imply for our models? Since the rotation rates are larger in relativistic gravity than in the Newtonian case for the same initial conditions, we expect that the criteria for the development of dynamic and secular triaxial instabilities are fulfilled in more models and for longer time intervals in relativistic gravity. To see whether this statement is correct we consider the evolution of the rotation rate $\beta_{\text {rot }}$ for models A2B4G1, A4B4G4 and A4B5G4 (see Fig. 15). In all these models $\beta_{\text {rot }}$ reaches its maximum value at the time of bounce. If the core experiences multiple bounces, this is reflected in multiple peaks of $\beta_{\text {rot }}$ (see upper panel). As expected, the rotation rate in the relativistic models exceeds that of the corresponding Newtonian ones. This holds both for the maximum value and the average value of the rotation rate.

Using the Newtonian thresholds $\beta_{\text {rot sec }}$ and $\beta_{\text {rot dyn }}$ (see discussion above) to judge whether a model is prone to developing triaxial instabilities, we see that relativistic effects do not cause an important change in the case of model A2B4G1 (upper panel of Fig. 15), because $\beta_{\text {rot }}>\beta_{\text {rot sec }}$ for less than $10 \mathrm{~ms}$. On the other hand, the behaviour of the rapidly rotating model A4B4G4 (middle panel) is quite different. Its rotation rate exceeds $\beta_{\text {rot sec }}$ near bounce time and remains above that value after core bounce in relativistic gravity (even exceeding $\beta_{\text {rot dyn }}$ for a short time), while in Newtonian gravity it falls below $\beta_{\text {rot sec }}$ again after ring-down. Since relativistic effects tend to lower the threshold value $\beta_{\text {rot sec }}$ in case of the gravitational radiation-driven instability (see discussion above), this model demonstrates that Newtonian simulations can yield incorrect predictions about the development of secular instabilities. On the other hand, in the extremely rapidly and differentially rotating model A4B5G4 (lower panel), secular instabilities are likely to develop in both Newtonian and relativistic gravity. 
However, instabilities on a dynamic timescale should only appear in the latter case, where $\beta_{\text {rot }}>\beta_{\text {rot dyn }}$ for almost $4 \mathrm{~ms}$. We note that for a neutron star with an average density of $\sim \rho_{\text {nuc }}$ and a radius of $\sim 20 \mathrm{~km}$, the dynamical timescale (= sound crossing timescale) is roughly $1 \mathrm{~ms}$.

Note that in this discussion $\beta_{\text {rot }}$ is the ratio of rotational energy to potential energy of the entire core. Strictly speaking, the criteria for triaxial instabilities should only be evaluated for the rotation rate $\beta_{\text {rot ic }}$ of the inner core (which eventually becomes the compact remnant), because the outer core is a separate entity as far as the dynamics is concerned. However, due to the rapid drop of density and rotation velocity observed in all models at the outer boundary of the inner core, the outer core contributes only little to the total rotation rate. Thus, we can safely assume that $\beta_{\text {rot ic }} \approx \beta_{\text {rot }}$. This behavior has also been observed in the Newtonian core collapse simulations of Zwerger (1995).

Despite the knowledge of the evolution of $\beta_{\text {rot }}$ during core collapse, predictions about the actual development of triaxial instabilities are difficult. The thresholds originate from a linear stability analysis and involve restrictions (e.g. homogeneous density, uniform rotation, etc.). Unfortunately, we cannot simulate the actual nonlinear growth of triaxial instabilities, because our code is restricted to axisymmetric flows. Therefore, we have begun to extend our code to three spatial dimensions. The results of the 3D simulations planned to be performed with the extended code will be presented in a future publication. Still, at present our results may help to predict the behavior and consequences of $\beta_{\text {rot }}$ of fully three-dimensional models (Houser et al. 1994; Smith et al. 1996; Rampp et al. 1998).

For large values of $\beta_{\text {rot }}$ the neutron star is highly nonspherical. In this regime we expect deviations of the CF metric from the exact metric in the range of a few percent (see Sect. 5.5 in Paper I). Additionally, in the equation for $\beta_{\text {rot }}$ (see Sects. 1 and 5.4 in Paper I), the potential energy $E_{\text {pot }}$ is determined by the (small) difference between the gravitational mass $E_{\text {grav }}$ and proper mass $E_{\text {proper. }}$ Even for strongly gravitating systems like neutron stars, this difference is only about $10 \%$ of the individual terms. Thus, if the values for $E_{\text {grav }}$ and $E_{\text {proper }}$ are subject to an error of a few percent in the CFC approximation, we expect $\beta_{\text {rot }}$ to deviate by several $10 \%$ relative to its value in an exact spacetime. In simulations of rapidly rotating neutron stars in equilibrium we could observe relative deviations between $\beta_{\text {rotexact }}$ and $\beta_{\text {rot CFC }}$ of about $15 \%$. Owing to the uncertainties with the stability thresholds and the calculation of the rotation rate in the $\mathrm{CFC}$ approximation, the above predictions from the evolution of $\beta_{\text {rot }}$ for the actual development of triaxial instabilities should be regarded as an estimate.

\section{Gravitational wave emission}

\subsection{Gravitational wave energy spectrum and emission}

A detailed discussion of the spectra of gravitational waves emitted during Newtonian rotational core collapse, and their dependence on the collapse type and dynamics can be found in the work of Zwerger \& Müller (1997). In the quadrupole approximation and in axisymmetry, the gravitational radiation field is solely determined by the amplitude $A_{20}^{\mathrm{E} 2}$ (see Appendix A). The total energy radiated away by gravitational waves is then given by

$E_{\text {rad tot }}=\frac{1}{32 \pi} \int_{-\infty}^{\infty}\left|\frac{\mathrm{d} A_{20}^{\mathrm{E} 2}}{\mathrm{~d} t}\right|^{2} \mathrm{~d} t$.

By replacing the gravitational wave amplitude in the time domain $A_{20}^{\mathrm{E} 2}(t)$ by its Fourier transform in the frequency domain $\hat{A}_{20}^{\mathrm{E} 2}(v)$, the total radiated energy $E_{\text {rad tot }}$ can be expressed as a frequency integral:

$E_{\text {rad tot }}=\frac{1}{16 \pi} \int_{0}^{\infty} v^{2}\left|\hat{A}_{20}^{\mathrm{E} 2}\right|^{2} \mathrm{~d} \nu$.

The spectral energy distribution is then

$\frac{\mathrm{d} E_{\mathrm{rad}}}{\mathrm{d} \nu}=\frac{1}{16 \pi} v^{2}\left|\hat{A}_{20}^{\mathrm{E} 2}\right|^{2}$

As a characteristic spectral measure of the gravitational wave signal we define the frequency $v_{\max }$ at which the energy spectrum $\mathrm{d} E_{\text {rad }} / \mathrm{d} v$ has its maximum (see also Zwerger \& Müller 1997).

As discussed in Sect. 3.2, the average and peak central densities of our relativistic models are larger than those of the Newtonian ones. This leads to an increase of the oscillation frequencies of the inner core during the ring-down phase in a regular collapse situation, and to a decrease of the time interval between consecutive bounces in multiple bounce models. Therefore, the gravitational wave spectrum is shifted towards higher frequencies in the relativistic models. This shift can be as large as a factor 5 in frequency (see Table 1 and lower panel of Fig. 16). The models with the largest frequency shift are those where the deeper relativistic gravitational potential changes a multiple bounce collapse into a regular collapse (see Sect. 3.3). We find that in Newtonian gravity only three models have a higher characteristic frequency $v_{\max }$ than the corresponding relativistic ones. However, the energy spectra of these models show many local maxima near $v_{\max }$ having almost the same spectral energy density. Fitting the energy spectra of the three models by a smooth function yields again a higher value of $v_{\max }$ in case of relativistic gravity.

The energy spectra of three models are plotted in Fig. 16. They are obtained by Fourier transforming the gravitational signal. A Welch filter is applied in the Fourier transformation to reduce the noise caused by the finite length of the wave signal (Press et al. 1992). To compensate for the power loss due to filtering, the spectrum is rescaled by the requirement that the total energy in the time domain, Eq. (3) equals that of the frequency domain, Eq. (4). The energy spectra are similar for both relativistic and Newtonian simulations in cases where the collapse type does not change (see upper and middle panel in Fig. 16). On the other hand, when a Newtonian model exhibiting multiple bounces changes to a regular collapse model in relativistic gravity, like e.g. model A3B3G1, the shape of its energy spectrum is shifted significantly (see lower panel in Fig. 16).

By integrating the spectral energy distribution, Eq. (5), over all frequencies, one obtains the total energy radiated away by the system in gravitational waves, as given in Eq. (4). However, 


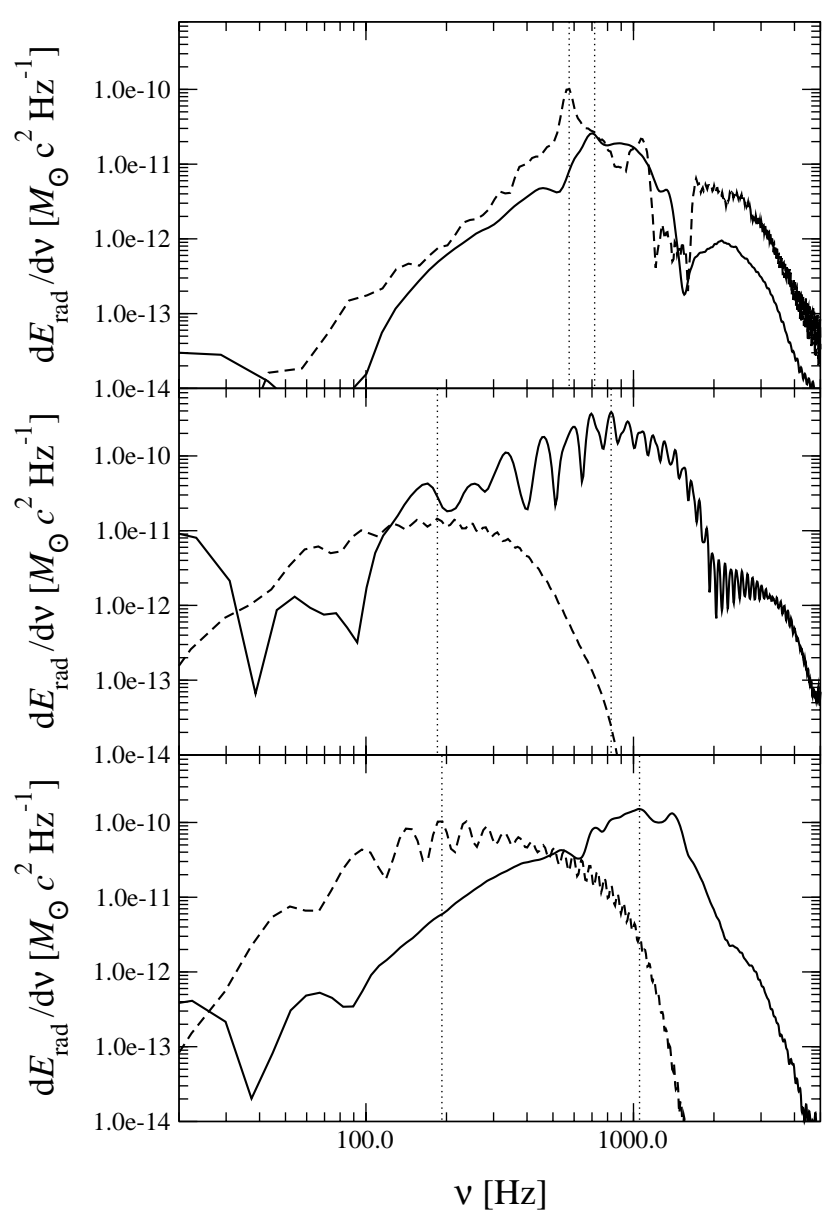

Fig. 16. Spectral energy distribution $\mathrm{d} E_{\text {rad }} / \mathrm{d} v$ of the gravitational wave signal in the relativistic (solid lines) and Newtonian (dashed lines) simulations of models A1B3G3 (upper panel), A3B3G1 (middle panel) and A1B3G1 (lower panel). The energy spectrum of model A1B3G3, with a strong low frequency part and a weaker high frequency part, is typical for the signal of a regular collapse. Model A3B3G1 is a multiple bounce collapse model in both Newtonian and relativistic gravity. In its energy spectrum a broad low frequency peak dominates. In model A1B3G1 relativistic effects change the collapse type from multiple bounce collapse to regular collapse. The energy spectrum reflects this change. The left (right) vertical dotted lines mark the maxima of the energy spectrum in Newtonian (relativistic) gravity. Due to higher average central densities in the relativistic simulations, the maximum of the energy spectrum is shifted to higher frequencies.

when analyzing numerical data this method is inappropriate, because high frequency noise contributes significantly to $E_{\text {rad tot }}$. For most models it is impossible to unambiguously define a cutoff frequency beyond which there exists only noise, as $E_{\text {rad }}$ does not drop sharply at some specific frequency. On the other hand, applying a filter (like the Welch filter used in Fig. 16) causes a leakage of spectral energy density, and thus leads to a underestimate of $E_{\text {rad tot }}$. Therefore, we use Eq. (3) to calculate $E_{\text {rad tot }}$.

We find that the total energy (measured in units of $M_{\odot} c^{2}$ ) radiated in form of gravitational waves lies in the range $3.0 \times$ $10^{-10} \leq E_{\text {rad tot }} \leq 3.7 \times 10^{-7}$ for the relativistic models, and in the range $3.8 \times 10^{-10} \leq E_{\text {rad tot }} \leq 1.5 \times 10^{-7}$ for the Newtonian ones. Averaged over all models, $E_{\text {rad tot }}=8.2 \times 10^{-8}$ in the relativistic case, and $E_{\text {rad tot }}=3.6 \times 10^{-8}$ in the Newtonian one, which corresponds to an increase of $128 \%$ due to relativistic effects.

\subsection{Gravitational wave amplitudes and frequencies}

The distribution of the dimensionless signal amplitude seen by an observer located in the equatorial plane of the source, i.e. the gravitational wave strain (see Eq. (A.3) with $\theta=\pi / 2$ )

$$
\begin{aligned}
h^{\mathrm{TT}} & =\frac{1}{8} \sqrt{\frac{15}{\pi}} \frac{A_{20}^{\mathrm{E} 2}}{r} \\
& =8.8524 \times 10^{-21}\left(\frac{A_{20}^{\mathrm{E} 2}}{10^{3} \mathrm{~cm}}\right)\left(\frac{10 \mathrm{kpc}}{r}\right),
\end{aligned}
$$

is shown in Fig. 17 for all 26 models, scaled to a distance of $r=10 \mathrm{kpc}$ from the source. The corresponding wave amplitudes $A_{20}^{\mathrm{E} 2}$ are listed in Table 1 .

A comparison of Fig. 17 with Fig. 8 in Zwerger \& Müller (1997) proves that our sample, encompassing 25 of the 78 models considered in the parameter study of Zwerger \& Müller (1997) - we have simulated the additional model A3B2G4 $4_{\text {soft }}-$ covers almost the same range of wave amplitudes $\left(4 \times 10^{-21} \leq\right.$ $\left.h^{\mathrm{TT}} \leq 3 \times 10^{-20}\right)$ and frequencies $(60 \mathrm{~Hz} \leq v \leq 1000 \mathrm{~Hz})$. Thus, relativistic effects have no significant impact on the gross distribution of expected signals in the amplitude-frequency diagram. Only in the high frequency, low amplitude region of the diagram, i.e. for models which collapse rapidly and have type I or III waveforms, the weaker signal amplitudes cause a discernible change in the model distribution. The bulk of the models falls into the range of detectability of the LIGO I interferometer (Gustafson et al. 1999) provided the source is located at a distance of less than $10 \mathrm{kpc}$ (see Fig. 17).

For all models which are of the same type in both Newtonian and relativistic gravity, the gravitational wave signal has a lower amplitude. When selecting those 13 models which are of type II in Newtonian gravity (see Sect. 3.1.2), the influence of relativistic effects on the signal amplitude is not as clearcut. The two relativistic models which remain of type II (see Table 1) yield smaller maximum amplitudes $\left|A_{20}^{\mathrm{E} 2}\right|_{\max }$. If the collapse type changes due to the higher central densities reached in relativistic gravity, some models exhibit significantly higher signal amplitudes than in Newtonian gravity (e.g. $+84 \%$ for model A1B3G1), whereas others possess smaller signal amplitudes (e.g. $-22 \%$ for model A3B1G1). In models which change their collapse type, the increase or decrease of the signal strength, and thus the change of location in the amplitude-frequency diagram, depends very strongly on the rotational state of the initial model and on the value of $\gamma_{1}$. However, as in all other models, relativistic gravity always causes a rise of the characteristic signal frequencies. 

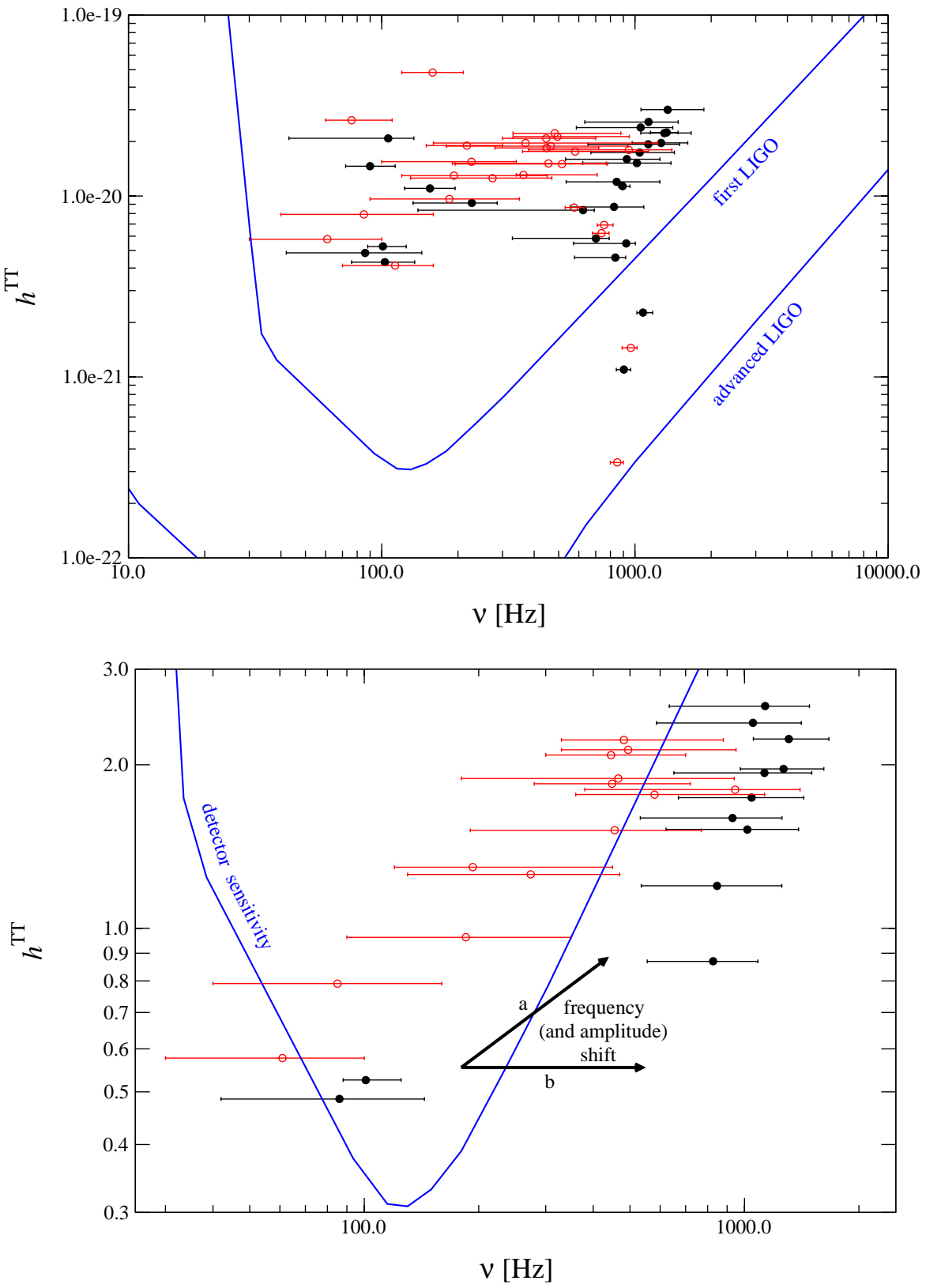

Fig. 17. Prospects of detection of the gravitational wave signal from axisymmetric rotational supernova core collapse in relativistic (black filled circles) and Newtonian (red unfilled circles) gravity. The figure gives the (dimensionless) gravitational wave amplitude $h^{\mathrm{TT}}$ and the frequency range for all 26 models. For a source at a distance of $10 \mathrm{kpc}$ the signals of all models are above the burst sensitivity of the LIGO I detector (except for some low amplitude, high frequency models), and well above that of the LIGO II interferometer. The burst sensitivity gives the rms noise amplitude $h_{\mathrm{rms}} \sim$ $\sqrt{v S(v)}$ over a bandwith of width $v$ at a frequency $v$ for the instrument noise power spectral density $S(v)$. The error bars mark the frequency range inside which the spectral energy density is within $50 \%$ of its peak value.

Fig. 18. Impact of the frequency and wave amplitude shift on the signal detectability for relativistic models which experience multiple bounces in Newtonian gravity. In the "best case", both amplitude and frequency increase in relativistic gravity (a). However, on average only the frequency increases (b). In neither case the shift is as steep as the high frequency sensitivity curve of an interferometer detector, and thus a relativistic model may fall out of the sensitivity window of the detector, if the Newtonian model is just within detection range. Note that the vertical scale is arbitrary. Symbols and error bars as in Fig. 17.
The overall behaviour of the 13 models which are of type II in Newtonian gravity, can be summarized by considering the arithmetic mean of their peak amplitudes and peak frequencies:

$$
\begin{aligned}
& \left.\begin{array}{l}
v_{\text {mean }}^{\mathrm{N}}=394 \mathrm{~Hz} \\
v_{\text {mean }}^{\mathrm{R}}=930 \mathrm{~Hz}
\end{array}\right\}+136 \%, \\
& \left.\begin{array}{l}
h_{\text {mean }}^{\mathrm{TT} \mathrm{N}}=1.55 \times 10^{-20} \\
h_{\text {mean }}^{\mathrm{TT} \mathrm{R}}=1.69 \times 10^{-20}
\end{array}\right\}+9 \% .
\end{aligned}
$$

Our simulations show that while the average gravitational wave signal amplitude $h^{\mathrm{TT}}$ does not change significantly, the frequency more than doubles.

This large frequency shift can have important consequences for the prospects of detection of gravitational waves. If the signal of a particular model is close to the detection threshold of a detector, as shown in Fig. 18 for the 13 models which experience multiple bounces in Newtonian gravity, the influence of relativity can be twofold. In the "best case scenario" the signal is shifted to both higher frequencies and amplitudes (case a). However, more frequently the location of the model will only shift towards higher frequencies in the amplitude-frequency diagram (case b - see Eq. (7)), and therefore in a borderline case the signal may actually leave the sensitivity window of the detector.

\section{Summary and conclusions}

We have presented hydrodynamic simulations of relativistic rotational supernova core collapse in axisymmetry and have analyzed the gravitational radiation emitted by such an event. We have simulated the evolution of 26 models in both Newtonian and relativistic gravity. Collapse of the initial configurations, 
which are differentially rotating relativistic 4/3-polytropes in equilibrium, is induced by decreasing the adiabatic index to some prescribed fixed value $\gamma_{1}$ with $1.28 \leq \gamma_{1} \leq 1.325$. The stiffening of the equation of state at nuclear matter density and the thermal pressure in the matter heated by the prompt shock are simulated by means of a simplified equation of state consisting of a polytropic and a thermal part. Microphysics like electron captures and neutrino transport is neglected.

Our simulations show that the three different types of rotational supernova core collapse and gravitational waveforms identified in previous Newtonian simulations (regular collapse, multiple bounce collapse, and rapid collapse) are also present in relativistic gravity. We find, however, that rotational core collapse with multiple bounces, which occurs when the collapse is only or predominantly stopped by centrifugal forces, is possible only in a much narrower parameter range in relativistic simulations. Only two of the 26 models of our sample show unambiguous multiple bounces in relativistic gravity. The peak central densities associated with the individual bounces in such models are much larger (up to a factor of 8 ) in the relativistic models compared to the corresponding Newtonian ones. Consequently, the time elapsed between subsequent bounces decreases by a factor of up to 4 .

We further find that relativistic gravity can have a qualitative impact on the dynamics. If the density increase due to the deeper relativistic potential is sufficiently large, a collapse which is stopped by centrifugal forces at subnuclear densities (and thus undergoes multiple bounces) in a Newtonian simulation, becomes a regular, single bounce collapse in relativistic gravity. A collapse type transition also has important consequences for the maximum gravitational wave signal amplitude.

For all relativistic models the peak density at bounce is larger than in the corresponding Newtonian models, the relative increase reaching $\sim 700 \%$ in some cases. This also holds for the maximum density of the compact remnant after ringdown in case of instant formation of a stable equilibrium state (types I and III). Nevertheless, we find that only six models have also larger maximum signal amplitudes than their Newtonian counterparts. The maximum signal amplitudes of all other relativistic models are (up to 57\%) smaller than those of the corresponding Newtonian ones. The reduced maximum signal strength can be explained by the fact that the quadrupole amplitude is determined by the bulk motion of the core rather than just by the motion of the densest mass shells. Therefore, a core which is more condensed in the center can give rise to a smaller gravitational wave signal than a core which is less centrally condensed, but which is denser and moves faster in its outer regions. However, the fact that all relativistic models show an increased central and reduced peripheral density of the collapsed core relative to their Newtonian counterparts does not necessarily imply a weaker gravitational wave signal, as in 6 of 26 relativistic models the maximum gravitational wave amplitudes are larger than in the corresponding Newtonian models. In all these six cases the collapse changes from type II (or type I/II) to type I when changing from Newtonian to relativistic gravity.

Overall, the relativistic models cover almost the same range of gravitational wave amplitudes $\left(4 \times 10^{-21} \leq h^{\mathrm{TT}} \leq 3 \times 10^{-20}\right)$ and frequencies $(60 \mathrm{~Hz} \leq v \leq 1000 \mathrm{~Hz})$ as the corresponding Newtonian ones. For all models which are of the same collapse type in both Newtonian and relativistic gravity, the gravitational wave signal is of lower amplitude. If the collapse type changes, either weaker or stronger signals are found in the relativistic case. Averaged over all models, the total energy radiated in form of gravitational waves is $8.2 \times 10^{-8} M_{\odot} c^{2}$ in the relativistic case, and $3.6 \times 10^{-8} M_{\odot} c^{2}$ in the Newtonian one.

For a given model, relativistic gravity can cause a large increase of the characteristic signal frequency of up to a factor of five. This frequency shift is an effect common to all models, and can have important consequences for the prospects of detection of gravitational waves. If the signal of a particular model is close to the high-frequency detection threshold of a detector, the influence of relativity can be twofold. In the "best case scenario" both the signal frequency and amplitude increase; however, more often only the signal frequency increases. In either case, in a borderline situation the signal may actually leave the sensitivity window of the detector, as the high-frequency detection threshold of a laser interferometer rises steeply with increasing frequency. Hence, within the limits of our physical model of rotational core collapse, relativistic effects on average tend to decrease the prospects of detectability of gravitational radiation by interferometric detectors. Nevertheless, the gravitational wave signals obtained in our study are within the sensitivity range of the first generation laser interferometer detectors if the source is located within the Local Group.

In our study we have restricted ourselves to simplified supernova core models. The complicated microphysics (electron captures and other weak interactions, equation of state) and neutrino transport processes have all been absorbed in one model parameter $\left(\gamma_{1}\right)$. Although this is an extreme simplification, we nevertheless think that it does not change the qualitative nature of our results. In particular, we do not expect that more realistic (axisymmetric) core collapse models will produce significantly stronger gravitational wave signals. Larger initial rotation rates and lower values of the (effective) adiabatic index $\gamma_{1}$, which could lead to stronger gravitational wave signals, are most likely excluded. This is also supported by the fact that the signal strengths of the microscopically more realistic, but Newtonian models of Mönchmeyer et al. (1991) agree well with those of our simplified models. To check whether these expectations are indeed correct we plan to incorporate a realistic equation of state and a simplified treatment of neutrino transport into our relativistic code in the near future.

In several models the rotation rate of the compact remnant exceeds the critical value where MacLaurin spheroids become secularly, and in some cases even dynamically unstable against triaxial perturbations $\left(\beta_{\text {rot sec }} \approx 13.8 \%\right.$ and $\left.\beta_{\text {rot dyn }} \approx 27.4 \%\right)$. It has been have pointed out that if such instabilities indeed occur, a much stronger gravitational wave signal might be produced than by cores which remain axisymmetric (for a review, see e.g. Thorne 1995). According to this idea, the core will be transformed into a bar-like configuration that spins end-over-end like an American football. One has further speculated, whether the core might even break up into two or more massive pieces,

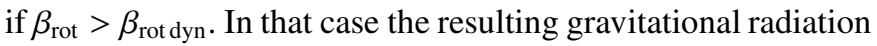
could be almost as strong as that from coalescing neutron star 
binaries (Thorne 1995). The strength of the gravitational signal sensitively depends on what fraction of the angular momentum of the non-axisymmetric core goes into gravitational waves, and what fraction into hydrodynamic waves. These sound and shock waves are produced as the bar or lumps, acting like a twirling-stick, plow through the surrounding matter. In order to investigate these effects, three-dimensional relativistic simulations of rotational core collapse have to be performed, which can follow the growth of non-axisymmetric instabilities (bar modes and gravitational wave driven unstable inertial modes). To this end we are currently extending our code to three spatial dimensions.

Acknowledgements. This work was finished during a visit of H.D. at the Universidad de Valencia, Spain. He would like to thank for the hospitality at the Departamento de Astronomía y Astrofísica. J.A.F. acknowledges support from a Marie Curie fellowship by the European Union (MCFI-2001-00032). All computations were performed on the NEC SX-5/3C supercomputer at the Rechenzentrum Garching.

\section{Appendix A: Gravitational wave extraction}

In the appendix we summarize the technical aspects related to the gravitational wave extraction and the different methods we have used. As stated in Paper I, the use of a CF metric implies the removal of the gravitational radiation degrees of freedom. Therefore, in order to compute the waveforms we apply the Newtonian quadrupole formula.

In that approximation and for an axisymmetric source in spherical coordinates, it can be shown (Thorne 1980) that the transverse traceless gravitational field has one independent component $h_{\theta \theta}^{\mathrm{TT}}$, which depends only on the quadrupole signal amplitude $A_{20}^{\mathrm{E} 2}$,

$h_{\theta \theta}^{\mathrm{TT}}=\frac{1}{r} A_{20}^{\mathrm{E} 2}(t-r) T_{\theta \theta}^{\mathrm{E} 220}$.

As the $l=2, m=0$ spherical tensor harmonic $T_{\theta \theta}^{\mathrm{E} 220}$ is defined according to

$T_{\theta \theta}^{\mathrm{E} 220}=\frac{1}{8} \sqrt{\frac{15}{\pi}} \sin ^{2} \theta$

the quadrupole radiation field $h_{\theta \theta}^{\mathrm{TT}}$ is given by

$h_{\theta \theta}^{\mathrm{TT}}=\frac{1}{8} \sqrt{\frac{15}{\pi}} \sin ^{2} \theta \frac{A_{20}^{\mathrm{E} 2}}{r}$.

\section{A.1. Different formulations of the quadrupole formula}

The amplitude $A_{20}^{\mathrm{E} 2}$ in Eq. (A.3) is the second time derivative of the mass quadrupole moment of the source,

$A_{20}^{\mathrm{E} 2}=\frac{\mathrm{d}^{2}}{\mathrm{~d} t^{2}}\left(k \int r^{4} \mathrm{~d} r \mathrm{~d} z \rho\left(\frac{3}{2} z^{2}-\frac{1}{2}\right)\right)$,

where $z=\cos \theta$, and $k=16 \pi^{3 / 2} / \sqrt{15}$. This formulation of the radiation field is known as the standard quadrupole formula.

A direct numerical implementation of this formula is problematic, as the discretization of the second time derivative causes high frequency noise in the gravitational wave signal.
The amplitude of this noise can be larger than the total amplitude of the signal. The problem is worsened by the fact that the density distribution in the integrand of Eq. (A.4) is weighted by $r^{4}$, i.e. mass elements in the outer parts of the star - where the grid resolution is coarser - contribute strongly to the signal.

To overcome this problem a standard practice is to replace the time derivatives by spatial derivatives (Finn 1989; Blanchet et al. 1990). This procedure leads to the stress formula for the gravitational wave amplitude:

$$
\begin{aligned}
A_{20}^{\mathrm{E} 2}= & k \int r^{2} \mathrm{~d} r \mathrm{~d} z \rho\left(v_{r}^{2}\left(3 z^{2}-1\right)+v_{\theta}^{2}\left(2-3 z^{2}\right)-v_{\varphi}^{2}\right. \\
& \left.-6 v_{r} v_{\theta} z \sqrt{1-z^{2}}-r \frac{\partial \Phi}{\partial r}\left(3 z^{2}-1\right)+3 \frac{\partial \Phi}{\partial \theta} z \sqrt{1-z^{2}}\right),
\end{aligned}
$$

where $\Phi$ is the Newtonian gravitational potential. This modified formulation of the quadrupole formula was used in our analysis.

\section{A.2. Ambiguity of the stress formula in the CFC approximation}

In a mathematically strict sense the quadrupole approximation of the radiation field, Eq. (A.1), is only defined for a flat Minkowski spacetime where the gravitational waves propagate as a linear perturbation. However, the multipole amplitudes and the tensor harmonics, although defined in flat spacetime, contain information about the wave source, which is situated in a strong field and high velocity region. In our simulations the quadrupole moment $A_{20}^{\mathrm{E} 2}$ is computed as a spatial integral over the relativistic matter configuration in a curved spacetime, as the metric $g_{\mu \nu}$ is not Minkowskian. Therefore, our methods to compute the radiation field are hampered by several ambiguities in the quadrupole formula.

Whereas in the Newtonian formulation, which is based on Euclidean geometry, the definition of the radius coordinate $r$ is no source of ambiguity, in the Riemannian geometry of a curved spacetime the circumferential radius and the coordinate radius do not necessarily coincide. In our simulations we use the isotropic radial coordinate $r$ in the computation of the quadrupole moment. Tests where we replaced $r$ by the circumferential radius in the wave extraction produced almost identical waveforms.

The spatial derivatives of the Newtonian potential present in Eq. (A.5) need particular attention. When simply using Poisson's equation,

$\Delta \Phi_{1}=4 \pi \rho$,

which we solve by an expansion into Legendre polynomials (Zwerger 1995), the waveform shows an offset, particularly after core bounce (see the dashed-dotted line in Fig. A.1). For strong gravity, the Newtonian potential $\Phi_{1}$ is therefore inappropriate for describing the radiation field. Another manifestation of this problem is a large constant offset in $A_{20}^{\mathrm{E} 2}$ for rapidly rotating neutron stars in equilibrium, where the gravitational radiation should vanish. 


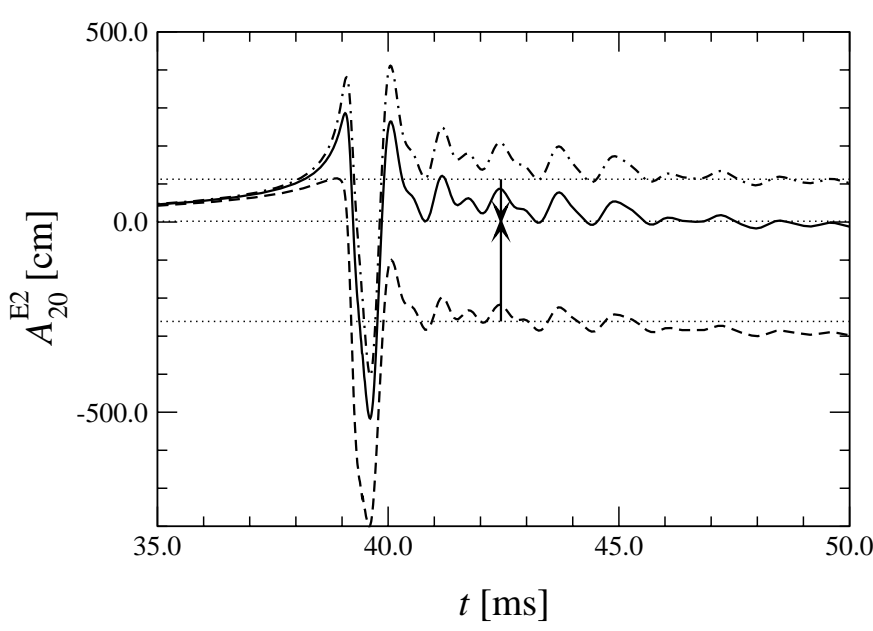

Fig. A.1. Reduction of the offset in the gravitational wave amplitude $A_{20}^{\mathrm{E} 2}$ for model A3B2G4 with the weighting method applied to the stress formula. If the Newtonian potential $\Phi_{1}$ is used, the wave signal shows a large positive offset (dashed-dotted line). If the combination of metric components $\Phi_{2}$ is used, a large negative offset occurs (dashed line). The weighted sum $\Phi_{\text {weighted }}$ of these two signals yields a waveform with practically zero offset (solid line). This combined signal exhibits no high frequency noise, as its constituents have been obtained using the stress formula.

The Newtonian potential can also be computed accurately up to the first post-Newtonian order by setting $g_{11} \approx(1-2 \Phi)$ equal to the conformal factor $\phi^{4}$. This leads to

$\Phi_{2}=\frac{1}{2}\left(1-\phi^{4}\right)$.

The waveforms obtained from this expression also show an offset, but this time a negative one (see the dashed line in Fig. A.1). However, combining both expressions for $\Phi$, Eqs. (A.6) and (A.7), into

$\Phi_{\text {weighted }}=\frac{\Phi_{1}+a \Phi_{2}}{1+a}$,

where $a=$ const. is a parameter with an appropriate value, one is able to reduce the offset almost to zero in all core collapse models (see the solid line in Fig. A.1).

\section{References}

Alcubierre, M., Brandt, S., Brügmann, B., et al. 2001, Int. J. Mod. Phys. D, 10, 273

Aloy, M. A., Müller, E., Ibáñez, J. M., Martí, J. M., \& MacFadyen, A. 2000, ApJ, 531, L119

Banyuls, F., Font, J. A., Ibáñez, J. M., Martí, J. M., \& Miralles, J. A. 1997, ApJ, 476, 221

Blanchet, L., Damour, T., \& Schäfer, G. 1990, MNRAS, 242, 289

Bodenheimer, P., \& Woosley, S. E. 1983, ApJ, 269, 281

Bonazzola, S., Frieben, J., \& Gourgoulhon, E. 1996, ApJ, 460, 379

Bonazzola, S., Frieben, J., \& Gourgoulhon, E. 1998, A\&A, 331, 280

Bonazzola, S., \& Marck, J. A. 1993, A\&A, 267, 623
Brown, J. D. 2001, Astrophysical sources for ground-based gravitational wave detectors, ed. J. M. Centrella, AIP Conf. Proc., 575, pp. 234-245 (American Institute of Physics, Melville, New York, USA)

Dimmelmeier, H., Font, J. A., \& Müller, E. 2001, ApJ, 560, L163

Dimmelmeier, H., Font, J. A., \& Müller, E. 2002, A\&A, 388, 917 (Paper I)

Evans, C. R. 1986, in Dynamical spacetimes and numerical relativity, ed. J. M. Centrella (Cambridge University Press, Cambridge, UK), pp. 3-39

Finn, L. S. 1989, in Frontiers in numerical relativity, ed. C. R. Evans, S. L. Finn, \& D. W. Hobill (Cambridge University Press, Cambridge, UK), pp. 126-145

Finn, L. S., \& Evans, C. R. 1990, ApJ, 351, 588

Fryer, C., \& Heger, A. 2000, ApJ, 541, 1033

Fryer, C., Holz, D. E., \& Hughes, S. A. 2002, ApJ, 565, 430

Goldreich, P., \& Weber, S. V. 1980, ApJ, 238, 991

Gustafson, E., Shoemaker, D., Strain, K., \& Weiss, R. 1999, LSC white paper on detector research and development, Technical Report LIGO T990080-00-D

Hayashi, A., Eriguchi, Y., \& Hashimoto, M. 1999, ApJ, 521, 376

Heger, A., Langer, N., \& Woosley, S. E. 2000, ApJ, 528, 368

Houser, J. L., Centrella, J. M., \& Smith, S. C. 1994, Phys. Rev. Lett., 72,1314

Imshennik, V. S., \& Nadezhin, D. K. 1992, Sov. Astron. Lett., 18, 79

Janka, H.-T., \& Mönchmeyer, R. 1989, A\&A, 226, 69

Janka, H.-T., Zwerger, T., \& Mönchmeyer, R. 1993, A\&A, 268, 360

Kaspi, V. M. 2000, in Pulsar Astronomy - 2000 and Beyond, M. ed. Kramer, N. Wex, \& N. Wielebinski (Astronomical Society of the Pacific, San Franciso, CA, USA), ASP Conf. Ser., 202, 485-490

Komatsu, H., Eriguchi, Y., \& Hachisu, I. 1989a, MNRAS, 237, 355

Komatsu, H., Eriguchi, Y., \& Hachisu, I. 1989b, MNRAS, 239, 153

Lorimer, D. R. 2001, Living Rev. Relativity 4, cited on 10 Dec 2001, http://www . livingreviews.org/Articles/Volume4/ 2001-5lorimer/

MacFadyen, A. I., Woosley, S. E., \& Heger, A. 2001, ApJ, 550, 410

Marshall, F., Gotthelf, E., Zhang, W., Middleditch, J., \& Wang, Q. 1998, ApJ, 499, L179

Mönchmeyer, R., \& Müller, E. 1989, in NATO ASI Timing neutron stars, ed. H. Ögelman, \& E. P. J. van den Heuvel (Kluwer, Dordrecht, Netherlands), pp. 549-572

Mönchmeyer, R., Schäfer, G., Müller, E., \& Kates, R. E. 1991, A\&A, 246, 417

Müller, E, 1982, A\&A, 114, 53

Müller, E. 1998, in Computational methods for astrophysical fluid flow, Saas-Fee Advanced Course 27, ed. O. Steiner, \& A. Gautschy (Springer, Berlin, Germany), pp. 343-494

Müller, E., \& Hillebrandt, W. 1981, A\&A, 103, 358

Müller, E., \& Janka, H.-T. 1997, A\&A, 317, 140

Nakamura, T. 1981, Prog. Theor. Phys., 65, 1876

Nakamura, T. 1983, Prog. Theor. Phys., 70, 1144

Nakamura, T., Oohara, K., \& Kojima, Y. 1987, Prog. Theor. Phys., 90,1

New, K. C. B., Centrella, J. M., \& Tohline, J. E. 2000, Phys. Rev. D, 62, 064019

Pradier, T., Arnaud, N., Bizouard, M.-A., et al. 2001, Phys. Rev. D, 63, 042002

Press, W. H., Teukolsky, S. A., Vetterling, W. T., \& Flannery, B. P. 1992, Numerical recipes in C: The art of scientific programming (Cambridge University Press, Cambridge, UK)

Rampp, M., Müller, E., \& Ruffert, M. 1998, A\&A, 332, 969 
Saijo, M., Shibata, M., Baumgarte, T. W., \& Shapiro, S. L. 2001, ApJ, 548, 919

Shapiro, S. L., \& Teukolsky, S. A. 1983, Black holes, white dwarfs, and neutron stars (Wiley, New York, USA)

Shibata, M. 2000, Prog. Theor. Phys., 104

Shibata, M., Baumgarte, T. W., \& Shapiro, S. L. 2000, ApJ, 542, 453

Smith, S. C., Houser, J. L., \& Centrella, J. M. 1996, ApJ, 458, 236

Stark, R. F., \& Piran, T. 1985, Phys. Rev. Lett., 55, 891

Stergioulas, N. 1998, Living Rev. Relativity 1, cited on 10 Dec. 2001, http://www. livingreviews.org/Articles/Volume1/ 1998-8stergio/

Stergioulas, N., \& Friedman, J. L. 1995, ApJ, 444, 306

Stergioulas, N., \& Friedman, J. L. 1998, ApJ, 492, 301

Symbalisty, E. M. D. 1984, ApJ, 285, 729

Tassoul, J.-L. 1978, Theory of rotating stars (Princeton University Press, Princeton, USA)

Thorne, K. S. 1980, Rev. Mod. Phys., 52, 299
Thorne, K. S. 1995, in Snowmass 1994 Summer Study on Particle and Nuclear Astrophysics and Cosmology in the next millennium, ed. E. W. Kolb, \& R. C. Peccei (World Scientific, Singapore), pp. $160-184$

Wheeler, J. C., Yi, I., Höflich, P., \& Wang, L. 2000, ApJ, 537, 810

Wilson, J. R., 1979, in Sources of gravitational radiation, ed. L. L. Smarr (Cambridge University Press, Cambridge, UK), pp. 423445

Wilson, J. R., Mathews, G. J., \& Marronetti, P. 1996, Phys. Rev. D, 54,1317

Yamada, S., \& Sato, K. 1994a, ApJ, 450, 245

Yamada, S., \& Sato, K. 1994b, ApJ, 434, 268

Yoshida, S., \& Eriguchi, Y. 1999, ApJ, 515, 414

Zwerger, T. 1995, Ph.D. Thesis, Technische Universität München, München, Germany

Zwerger, T., \& Müller, E. 1997, A\&A, 320, 209 